This dissertation has been microfilmed exactly as received

GRAHAM, Henry Collins, 1934-

PARTICLE SIZE DEPENDENCE OF THE ELECTRICAL CONDUCTIVITY OF NaCl.

The Ohio State ${ }^{\frac{1}{}}$ University, Ph.D., 1965

Physics, solid state

University Microfilms, Inc., Ann Arbor, Michigan 


\section{PARTICLE SIZE DEPENDENCE OF THE ELECTRICAL CONDUCTIVITY OF $\mathrm{NaCl}$}

\section{DISSERTATION}

\section{Presented in Partial Fulfillment of the Requirements for the Degree of Doctor of Philosophy in the Graduate School of The Ohio State University}

By

Henry Collins Graham, B.S., M.S.

The Ohio State University

1965

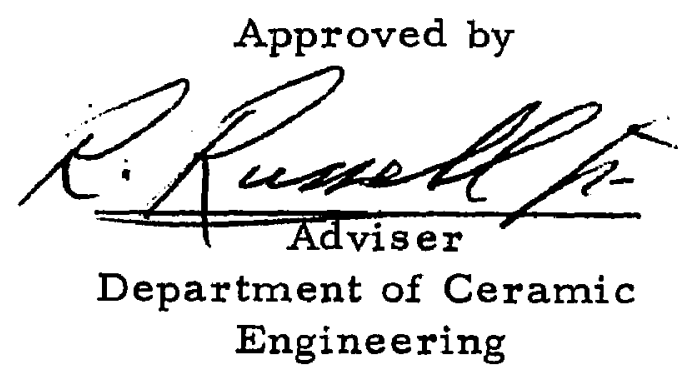




\section{ACKNOW LEDGMENTS}

The author wishes to express his appreciation to Professor Ralston Russell, Jr., adviser, for his help and encouragement throughout the course of this work and the graduate program. I am indebted to Dr. Norman M. Tallan for the suggestion of the problem and for his continuous and unstinting technical assistance.

I also wish to thank Dr. Robert W. Vest for many helpful discus sions, Dr. Harry A. Lipsitt for assistance in the particle size determinations, and Mr. James M. Wimmer for technical assistance throughout the work.

The outstanding support of the entire Metallurgy and Ceramics Research Laboratory, and particularly that of Mr. Elwood D. Wysong and $\mathrm{Mr}$. Harold G. Hoover, is gratefully acknowledged.

The support and self-sacrafice by my wife, Mary Lou, is sincerely appreciated.

I also wish to acknowledge the support of the United States Air Force through which I obtained both the necessary course work for my degree and the time and facilities for my dissertation. 
VITA

August 16, 1934

1956 ....

$1958 \ldots . .$.

$1958-1960 \ldots$

$1960-1965$...
Born - Pittsburgh, Pennsylvania

B.S., New York State College of Ceramics at Alfred University, Alfred, New York

M. S. , New York State College of Ceramics at Alfred University, Alfred, New York

Research Engineer, Pittsburgh Plate Glass Research Center, Harmarville, Pennsylvania Research Physicist, United States Air Force, Wright-Patterson Air Force Base, Ohio 
CONTENTS

Page

I. INTRODUCTION ................. 1

A. General ................. 1

B. Cation conductivity ............. 2

1. Intrinsic range ............. 2

2. Extrinsic range........... 3

C. Anion conductivity. ............ 4

D. Time-dependent conductivity .......... 9

II. ÁPPARATUS AND PROCEDURE ........... 12

A. Sample preparation ............ 12

1. Single crystal............. 12

2. Polycrystalline............. 12

3. Electrodes ............... 14

4. t/A Determinations........... 15

B. Sample holder............... 16

C. Sample environment. ........... 20

D. Measuring equipment........... 20

1. General ............. 20

2. d. c. Conductivity............. 24

3. a. c. Conductivity .............. 30

4. Readout .............. . 32

5. Density determinations.......... 32

6. Additive coloring ........... 33 
III. RESULTS AND DISCUSSION. . . . . . . . . . 35

1. Single crystal, as-received ........ 35

2. Polycrystalline, non-doped. ........ 39

3. Single crystal, additively colored ...... 51

IV. SUMMARY ................. 62 APPENDIX........................ 64 BIBLIOGRAPHY .................... 79 


\section{ILLUSTRATIONS}

Figure

1. Sample Holder. . . . . . . . . . . . . 17

2. Close-Up of Sample in Sample Holder . . . . . . . 18

3. Simplified Drawing of Sample Holder. . . . . . . 19

4. Schematic of Environment Control Equipment . . . . 21

5. Furnace Assembly . . . . . . . . . . . . 22

6. Block Diagram of Measuring Equipment . . . . . . 23

7. Wiring Diagram of Polarization Equipment . . . . 25

8. Schematic of Polarization Circuit-Constant Voltage . 26

9. Schematic of Polarization Circuit-Constant Current . 27

10. A. C. Circuit Schematic ........... 31

11. Bomb Used to Additively Color $\mathrm{NaCl}$. . . . . . . . 34

12. Conductivity versus Reciprocal Temperature for "As-Received" Single Crystal Sample . . . . . . 36

13. Conductivity versus Reciprocal Temperature for Polycrystalline Sample + 100 Mesh. . . . . . . . 40

14. Conductivity versus Reciprocal Temperature for Polycrystalline Sample $-100+200$ Mesh. . . . .

15. Conductivity versus Reciprocal Temperature for Polycrystalline Sample $-200+325$ Mesh . . . . . . 42

16. Conductivity versus Reciprocal Temperature for Polycrystalline Sample $-325+400 \mathrm{Mesh}$. . . . . .

17. Conductivity versus Reciprocal Temperature for Polycrystalline Sample -400 Mesh. . . . . . . . 44 
18. Conductivity versus Reciprocal Temperature for Polycrystalline Sample - Jet Milled ........ 45

19. Summary of Polycrystalline Conductivity Results . . 47

20. Conductivity and Transference Number as a Function of Particle Size . . . . . . . . . . . 48

21. Conductivity versus Reciprocal Temperature for Additively Colored Single Crystal Sample - Short Treatment. . . . . . . . . . . 52

22. Conductivity versus Reciprocal Temperature for Additively Colored Single Crystal Sample - Medium Treatment.................. 53

23. Conductivity versus Reciprocal Temperature for Additively Colored Single Crystal Sample - Long Treatment. ................ 54

24. Logarithm of Defect Concentration versus the Logarithm of $\mathrm{PCl}_{2}{ }^{1 / 2}$ for $\mathrm{NaCl}$. . . . . . . 57 


\section{LIST OF TABLES}

Table

Page

1. Chemical and Spectrographic Analysis of Harshaw

Chemical Company NaCl. . . . . . . . . . 12

2. Particle Sizes and Densities of Polycrystalline Samples .................. 14

3. Data for "As-Received" Single Crystal Sample. . . . 64

4. Data for + 100 Mesh Polycrystalline Sample . : . . 66

5. Data for $-100+200$ Mesh Polycrystalline Sample. . . 67

6. Data for $-200+325$ Mesh Polycrystalline Sample. . . 69

7. Data for $-325+400$ Mesh Polycrystalline Sample. . . 71

8. Data for -400 Mesh Polycrystalline Sample. . . . . 72

9. Data for Jet Milled Polycrystalline Sample . . . . 73

10. Data for Additively Colored Single Crystal - Short Treatment ................. 75

11. Data for Additively Colored Single Crystal - Medium Treatment ................. 75

12. Data for Additively Colored Single Crystal - Long Treatment . . . . . . . . . . . . . . . 


\section{INTRODUCTION}

\section{A. General}

The ionic transport mechanisms of $\mathrm{NaCl}$ have been extensively studied due in large part to sodium chloride's relatively simple structure for a non-metal and to its convenient temperature range, which includes its melting point. The conductivity has been shown to be ionic with the

predominent carrier the free cation vacancy. ${ }^{1}$ The data have been divided into a high temperature "intrinsic" region and a low temperature "extrinsic" region. In the intrinsic region the concentration of the defects is the Schottky, thermal equilibrium concentration. The concentration of defects in the extrinsic region may be controlled by surface or grain boundary effects, aliovalent impurities present and/or the prior history of the specimen. In most cases the divalent cation impurity content is large enough ( $100 \mathrm{ppm}$ is generally sufficient) to completely control the $\mathrm{Na}$ ion vacancy concentration and thereby the conductivity in the extrinsic range.

In contrast to this fairly complete picture, the development of the understanding of the anion transportmechanism or mechanisms has been rather slow. This has been the result of the relatively more difficult experimental procedures encountered due to the $\mathrm{Cl}$ ion's much smaller contribution and to the lack of interest (also related to this small contribution). Tubandt, ${ }^{2}$ in some of the early work on the halides, determined that the contribution of the chlorine ion to the total conduction was less than $10 \%$ at high temperatures and that it was insignificant in the lower 
temperature extrinsic region. However, the more recent work of Laurent and Bénard ${ }^{3}$ showed an enhancement of chlorine diffusivity as the particle size of the compact was decreased. While it has been well established that the movement of the chlorine ion is preferentially in the grain boundary region, ${ }^{3,4}$ the identity of the defect or defects responsible is still uncertain even in the light of articles recently published. ${ }^{5,6,7}$ The demonstration by Cabane 4 that the mechanism of enhanced chlorine diffusivity is dependent upon the degree of perfection of the grain boundary still has not led to the identification of the responsible defect (vacancy pair or free chlorine vacancy) or its movement mechanism.

In an effort to understand better the available data on anion conductivity in $\mathrm{NaCl}$ and in particular the mechanism of enhancement this work was undertaken.

\section{B. Cation conductivity}

Many papers have been published concerning the transport properties of the $\mathrm{Na}$ ion in $\mathrm{NaCl}$ and there are good review articles on the subject. ${ }^{8}$ The purpose of this section is to establish the background for the work on the anion transport properties rather than to cover the available literature on cation transport.

1. Intrinsic range. As previously stated, the intrinsic range in $\mathrm{NaCl}$ is the region when the conductivity is controlled by the concentration of the thermally generated, Schottky disorder. The accepted activation energy for this range $(1.86 \pm 0.03 \mathrm{eV})$ is well established and is considered to be the sum of one-half the energy to form a Schottky defect and the energy to move the $\mathrm{Na}$ ion vacancy.

Dreyfus and Nowick ${ }^{10}$ compared the available diffusivity and conductivity data for the $\mathrm{Na}$ ion. They found that it was not necessary to subtract an anion contribution from the total conductivity for the total conductivity to agree with that calculated from diffusivity data using the Nernst Einstein equation. In the light of the $10 \%$ maximum anion 
contribution found by Tubandt this agreement is not surprising considering the experimental scatter generally found in diffusivity results. However, the results to be presented here indicate that the contribution of the chlorine ion may be considerable and cannot be ignored, especially for specimens of small particle size.

2. Extrinsic range. The extrinsic portion of the cation conductivity, as its classification implies, varies from sample to sample depending upon the influence of factors external to the NaCl lattice itself. While it is possible to have a nonequilibrium defect concentration at low temperatures by quenching the sample, the slow cooled, equilibrium, conductivity is generally broken into several distinct ranges.

The first extrinsic range encountered upon cooling is characterized by a constant concentration of cation vacancies which is fixed by the concentration of divalent cation impurities..$^{10}$ The considerable latitude in the magnitude of the activation energy reported for this range may be in large part due to its usually short temperature range. Dreyfus and Nowick ${ }^{10}$ considered $0.80 \mathrm{eV}$ to be their best value even though their results varied from $0.70 \mathrm{eV}$ to $0.83 \mathrm{eV}$. Since the concentration of cation defects is fixed in this range, this activation energy is for movement of the $\mathrm{Na}$ ion vacancy. Therefore, the difference between this energy and the intrinsic energy (the energy of movement plus $1 / 2$ the energy of formation) is $1 / 2$ the Schottky energy. This calculation-gives the accepted value of $2.12 \pm 0.06 \mathrm{eV}, 10$ which is in excellent agreement with the most reliable theoretical estimates ${ }^{11}[2(1.86 \pm 0.03-0.80 \pm 0.03)=$ $2.12 \pm 0.06]$

The second range encountered in the extrinsic region as the temperature is decreased is characterized by the association of the oppositely charged divalent cation impurities and the cation vacancies forming a neutral complex. The early onset of this association is respongiblefor the usually limited extent of the first extrinsic range as mentioned 
previously. The activation energy found for this region is the sum of the energy of movement of the cation vacancy and $1 / 2$ the energy of association of the complex (approximately $1.0 \mathrm{eV}$ ). 10

The third range encountered in slowly cooled samples is one attributed to the precipitation of the divalent-cation impurities. At still lower temperatures where appreciable precipitation is prevented by the kinetics involved, the concentration of defects is again controlled by the association of the cation impurities and the cation vacancies. In rapidly cooled samples the precipitation range may be eliminated, and the two association ranges then merge. While some measurements in this work were made at the low temperatures where these reactions occur, the pertinent work was done over the temperature range which included the intrinsic region and impurity controlled extrinsic region, i. e., generally at temperatures greater than $300^{\circ} \mathrm{C}$.

\section{Anion conductivity}

The existing literature on the movement of the anion in $\mathrm{NaCl}$ is far from conclusive with respect to either the nature of the defect or de fects or to the energies associated with that movement. While some early work ${ }^{12}$ indicated the presence of both an intrinsic and an extrinsic range, more recent work has been inclined to support a single range. 6,7 The later work implies that the measured diffusivities are the result of movement by 2 defects - the free anion vacancy and the anion-cation vacancy pair.

Harrison, Morrison and Rudham" found a "knee" in the log diffusivity vs. reciprocal temperature curve for anion diffusion. Lidiard ${ }^{5}$ suggested that their interpretation of these results, on the basis of a range where the concentration of anion vacancies was fixed by the divalent-anion impurities present, was incompatible with the law of mass action in the presence of positive divalent impurities. He further 
suggested that the observation could be interpreted on the basis of a contribution to the diffusivity by the movement of vacancy pairs. His suggested test of this hypothesis was to measure the anion diffusivity as a function of the concentration of divalent cation impurity. Moxrison and Rudham, ${ }^{13}$ working with $\mathrm{KCl}$, varied the positive impurity content and reported the $\mathrm{Cl}$ diffusion coefficient to be insensitive to the impurity concentration. Coupled with the recognized possibility of vacancy pairs in alkali halides and the estimate of Seitz ${ }^{14}$ that the concentration of pairs and free vacancies should be equal at $600^{\circ} \mathrm{C}$, this was strong evidence in favor of a significant contribution by vacancy pairs.

Laurance ${ }^{6}$ studied the affect of doping with $\mathrm{Ca}^{+}$on the diffusivity of the $\mathrm{Cl}$ ion in NaCl. While his results did show a decrease in diffusivity with increased $\mathrm{Ca}^{2}+$ content, the decrease was small compared to the calculated change expected from the decreased concentration of $\mathrm{Cl}^{-}$vacancies as required by the law of mass action for Schottky disorder. Also the diffusivity was insensitive to impurity content above a relatively small concentration. He interpreted these results for high temperatures as follows:

a. the predominent anion defect in the pure crystal is the free vacancy;

b. in the doped crystal, the vacancy pair predominates;

c. at small dopant concentrations a combination of the two will be seen.

His qualitative analysis, based on the results of a determination of the $\mathrm{Ca}^{++}$content by measuring the electrical conductivity, put the break over point - mixed to pair - at less than $10^{-5}$ mole fraction calcium/ sodium.

Using this classification, he analyzed the experimental activation energies found for the pure and the doped samples (2.12 and $2.49 \mathrm{eV}$ respectively). Assuming the single range found for the pure crystal to 
be intrinsic and using the value of $2.02 \mathrm{eV}$ for the Schottky energy, the energy calculated for movement of the anion vacancy was $1.11 \mathrm{eV}$. The analysis of the doped activation energy was more complicated since neither the energy for movement or the binding energy of the vacancy pair is known experimentally. Using Fumi and Tosi's ${ }^{15}$ calculated binding energy $(0.60 \mathrm{eV}$ or less for $\mathrm{NaCl})$ and 2.02 for the Schottky energy, the energy for movement of the vacancy pair was $1.07 \mathrm{eV}[2.49-12.02-$ $0.60)=1.07]$. Combining these very similar energies for motion with the calculated densities of the two defects ${ }^{5}$ gave diffusivities in good agreement with the experimental values.

Commenting upon the compatibility of the proposed neutral vacancy pair theory with the work of Barr et al.,$^{12}$ which showed a sensitivity to dislocation content for chlorine ion diffusion in $\mathrm{NaCl}$, Laurance stated that the interaction could depend only indirectly upon the charge of the dislocation and would rather have to affect the number or mobility of the neutral defect.

The possibility that the significant contribution of the dopant was to alter the dislocation content was also suggested.

The very recent work of Barr et al.' has presented additional evidence in favor of vacancy pairs making a significant contribution to the anion movement in $\mathrm{NaCl}$. By a process of iteration between the experimental data and the possible diffusion equations for the two proposed carriers, the experimental diffusion coefficient was divided into a contribution for free vacancy and one for vacancy pairs. The two equations were

$$
\begin{aligned}
& \mathrm{D}_{\mathrm{V}_{\dot{\mathrm{Cl}}}}=1.1 \exp (-1.92 / \mathrm{kT}) \\
& \left.\mathrm{D}_{\left(\mathrm{V}_{\mathrm{Na}}^{\prime}\right.} \mathrm{V}_{\mathrm{Cl} 1}^{\cdot}\right)^{\mathrm{x}}=363 \exp (-2.37 / \mathrm{kT}) \\
& \text { where } \left.\mathrm{D}_{\text {experimental }}=\mathrm{D}_{\mathrm{V}_{\mathrm{Cl}}}+\mathrm{D}_{\left(\mathrm{V}_{\mathrm{Na}}^{\prime}\right.} \mathrm{V}_{\mathrm{Cl}}\right)^{\mathrm{x}}
\end{aligned}
$$


The labeling convention of Kroger and $\mathrm{Vink}^{16}$ is used. For any given defect or ion, the subscript indicates the lattice position occupied by it and the superscript the charge relative to the lattice. The three charge states, positive, negative and neutral, are designated by $\cdot$, ', and $\mathbf{x}$, respectively. For example, in $\mathrm{NaCl}$ a divalent cation impurity on a normal monovalent cation site is designated thusly, $\mathrm{F}_{\mathbf{N a}}$ or $\mathrm{F}_{\dot{M}}$; an anion vacancy, $\mathrm{V}_{\mathrm{Cl}}$; a neutral anion vacancy, $\mathrm{V}_{\mathrm{Cl}} \mathbf{x}$ (an F-center); a cation vacancy, $\mathrm{V}_{\mathrm{Na}}^{\prime}$; etc.

The $1.92 \mathrm{eV}$ found for intrinsic free vacancy diffusivity produced $0.9 \pm 0.1$ as the energy for movement of the anion vacancy $\left(1.9-\frac{2.0}{2}\right.$ $=0.9 \mathrm{eV})$. And again, as with Laurance's results, the estimated energy for pair movement is slightly larger $(2.4-(2.0-0.6)=1.0 \mathrm{eV})$.

Large variations in the diffusivities of $\mathrm{Ca}^{+}$doped specimens with respect to their calcium content could not be satisfactorily explained and therefore the data could not be used to strengthen the conclusions. However, the results could be described by a single straight line and were in good enough agreement to support the earlier results of Laurance, who ascribed the doped crystal diffusivity to vacancy pair movement.

The autoradiographic and diffusivity study by Cabané ${ }^{4}$ made several contributions to the overall picture of anion diffusion with probably the most significant being that the quality of the grain boundary was very important with respect to the enhanced anion diffusion in alkali halides.

The following results of his work were in agreement with those published earlier by Laurent and Bérnard. ${ }^{3}$

1. Anion diffusion was enhanced in the grain boundary region for the alkali halides.

2. Cation diffusion was not.

3. For a given host alkali halide the larger the anion the easier its movement, i.e., in $\mathrm{KCl}$ or $\mathrm{NaCl}, \mathrm{I}^{-}$diffusivity is greater than $\mathrm{Cl}^{-1} \mathrm{~s}$.

The results of Barr et al. ${ }^{12}$ with respect to the effects of dislocations in sodium chloride, are very interesting and eventually they will 
probably help explain the enhancement mechanism. The fact that the activation energy increased and the diffusivity decreased as the dislocation density decreased, was very strong evidence in favor of the important part played in enhanced diffusion by dislocations.

In the transparent samples prepared by Cabarie by the elimination of moisture prior to pressing, the grain boundaries werefound to be extremely thin and of excellent quality. The densities were within $0.1 \%$ of theoretical. For these samples, even though radiographic measurement showed there to be an increase of anion diffusion in the vicinity of grain boundaries, the phenomenon cannot be observed at high temperatures by measuring the relative diffusivities since the ratio $D^{\prime} \delta / D\left(D^{\prime}\right.$ = intergranular anion diffusion, $\delta=$ grain boundary thickness and $\mathrm{D}=$ bulk anion diffusion) is small to begin with and decreases with increasing temperature. For the normal opaque sample from which the moisture had not been removed, the ratio $D^{\prime} \delta / D$ was large and practically constant with increasing temperature since the activation energies for diffusion in the bulk and in the grain boundary region are approximately equal. Cabańe found the following activation energies for $\mathrm{K} \mathrm{Cl}: \mathrm{E}_{\mathrm{bulk}}$ $=25.7 \mathrm{kcal} / \mathrm{mole}, E_{\text {intergranular }}=25 \pm 3 \mathrm{kcal} / \mathrm{mole}$ for opaque $\mathrm{sam}$ ples and $12 \pm 2 \mathrm{kcal} / \mathrm{mole}$ for transparent samples.

Both Cabanie $e^{4}$ and Laurent and Bénard ${ }^{3}$ explained the mechanism responsible for the enhancement of the anion only in the following very general way. In the bulk of the material the size of the ion determines its mobility, hence the smaller cation is the more mobile. In the grain boundary region the larger ions undergo an increase in mobility pos sibly related to their easier polarizability. No conclusions are drawn by Cabanie with respect to the decreased activation energy for the intergranular diffusion in the samples with very good grain boundaries.

The intentional doping of $\mathrm{NaCl}$ with a divalent anion impurity, $\mathbf{S}^{2-}$ for instance, would seem to be a very easy way to depress the cation vacancy concentration and thereby bring the anion vacancy concentration 
up to a level which would increase its conductivity to a measurable value. However, this type of doping has been tried by several investigators, including the author, without success. It is very difficult to maintain the sulfur in the lattice as a divalent ion.

Another means of depressing the cation vacancy concentration is to additively color a crystal. If $\mathrm{NaCl}$ is heated in the vapor of an alkali metal, F-centers will be produced, While the impurity defects are not well defined, it is possible that the presence of impurities will produce other color centers. However, the predominance of the F-center is well established. ${ }^{17}$ Using the de Boer model, ${ }^{18}$ the coloration equation is $^{19}$

$$
\mathrm{Na}_{\text {vapor }}^{\mathbf{x}} \rightarrow \mathrm{Na}_{\mathrm{Na}}^{\mathbf{x}}+\mathrm{V}_{\mathrm{Cl}}^{\mathbf{x}}
$$

While the F-center is neutral and cannot contribute to the electrical conductivity, it has been demonstrated that the migration of a color center front will take place under an applied d.c. fleld. The proposed mechanism is the thermal dissociation of the color center, the movement of the electron in one direction and the anion vacancy in the opposite direction (or possibly the cation vacancy in the same direction as the electron). The movement of the electrons to the anode is assumed to occur by a series of these dissociations and recombinations with available charged anion vacancies. The free electrons reaching the anode pass out of the crystal and thereby reduce the concentration of $F$ centers. Experimental observations ${ }^{20}$ suggest that the reduction of sodium ions to the free metal at the cathode maintains charge neutrality without the introduction of new color centers. Once the electrons are all removed by the d. c. field, the crystal remains colorless and contains an excess of anion vacancies.

\section{Time-dependent conductivity}

The fact that a material may become polarized in an electric field due to the blocking of mobile charged defects or the rotation of dipoles 
within it, has been known for some time. ${ }^{21}$ Using electrodes which are blocking to one of the charge carriers will allow the contribution of the non-blocked species to be determined. Knowing this, the transference number for the species may be calculated and used to determine the conductivity of each with respect to the total. Depending upon the ambient conditions and the material, this polarization mechanism may account for a large percentage of the total charge transport in the material.

In $\mathrm{NaCl}$ several polarization mechanisms have been seen. Inquenched samples, a dipole rotation polarization was found in the temperature range $-60^{\circ}$ to $0^{\circ} \mathrm{C}$ and was identified as the orientation of $\left(\mathrm{F}_{\mathrm{Na}} \mathrm{V}_{\mathrm{Na}}^{\prime}\right)^{x}$ complexes. ${ }^{22}$ In slow cooled crystals, dielectric relaxation measurements and internal friction measurements have been used to study a polarization mechanism at temperatures less than $300^{\circ} \mathrm{C}{ }^{23}$ The combined results have been used to identify it as the rotation of the same complex as seen above. Sutter and Nowick ${ }^{24}$ earlier had made detailed analyses of what was termed "two diverse theories" for explaining a polarization mechanism in this same range. They concluded that their results fit the dielectric relaxation theory and implied that the space charge polarization theory was not valid. However, as was pointed out by Jacobs and Maycock, ${ }^{25}$ in order to adequately interpret the polarization phenomena it is necessary to study the material over a wide range of temperatures. When this is done for $\mathrm{NaCl}$, a high temperature polarization contribution to the conductivity is found which does in fact fulfill the requirements of the space charge theory. (In this work both the $<300^{\circ} \mathrm{C}$ and the high temperature polarization were seen.)

Recent dielectric relaxation and potential probe results for a fast time polarization in $\mathrm{NaCl}$ at temperatures between 300 and $400^{\circ} \mathrm{C}$, have been interpreted on the basis of an interfacial polarization mechanism, i. e., a build up of charge at an interface between the bulk and a preexisting, very thin $(20-50 \mu)$ surface layer, the resistivity of which is approximately fifty times that of the bulk. ${ }^{26}$ While this mechanism still 
falls in the classification of a fast time process, it serves to point out the possibility of a fast time process being due to movement other than a simple dipole rotation. An approach for distinquishing between a dipole rotation mechanism and an interfacial polarization mechanism on the basis of the relative magnitudes of the peak heights and the capacitance or the conductivity frequency dispersions has been presented by Tallan and Graham. ${ }^{27}$

The high temperature polarization process seen for $\mathrm{NaCl}$ in this work is very similar to that seen in $\mathrm{KCl}$ by Allnatt and Jacobs.8, 29 The polarization in pure $\mathrm{KCl}$ was attributed to a build up of charge at an electrode due to the blocking of the cation at the electrode. ${ }^{29}$ While the intrinsic steady state conductivity $\left(\sigma_{\infty}\right)$ was not identified with respect to which charge carrier was responsible for it, the statement was made that the a. c. conductivity $\left(\sigma_{0}\right)$ was due to the summation of the conductivities of two carriers. (When plotted on a $\log \omega T$ vs $1 / T$ graph, the intrinsic a. c. conductivity was not a straight line.) It is interesting to note that the transference number of the chlorine ion in $\mathrm{KCl}^{2}$ was very similar to that determined for $\mathrm{NaCl}^{2}$ (small), but that the polarization was large in $\mathrm{KCl}$ as it will be shown to be for $\mathrm{NaCl}$. 


\section{APPARATUS AND PROCEDURE}

\section{A. Sample preparation}

1. Single crystal. A 100 gram $\mathrm{NaCl}$ single crystal boule from the Harshaw Chemical Company was the source of both the single and polycrystalline samples used in this work. The chemical and spectrographic analyses of the single crystal and the fractionated powder are shown in Table 1. The pieces needed for the single crystal measurements were cleaved from the boule prior to its being ground and fractionated.

Table 1. Chemical and Spectrographic Analysis of Harshaw Chemical Co. $\mathrm{NaCl}$

\begin{tabular}{|c|c|c|c|c|c|c|c|c|}
\hline \multirow{2}{*}{ Sample } & & & \multicolumn{6}{|c|}{ Element (ppm by weight) } \\
\hline & $\mathrm{Ba}$ & Si & $\mathrm{Mg}$ & $\mathrm{Fe}$ & $\mathrm{Al}$ & $\mathrm{Y}$ & $\mathrm{Cu}$ & $\mathrm{Ca}$ \\
\hline $\begin{array}{l}\text { single } \\
\text { crystal }\end{array}$ & $<5(N)$ & 5 & $<5(\mathrm{~N})$ & 5 & 5 & $<10(N)$ & 5 & $<5(T)$ \\
\hline $\begin{array}{c}\text { powder } \\
-100+200 \\
\text { mesh }\end{array}$ & $<5(N)$ & 5 & $<5(N)$ & 10 & 5 & 10 & 5 & $<5(T)$ \\
\hline
\end{tabular}

(N) Not found.

(T) Trace.

2. Polycrystalline. The first five powder fractions were prepared by mechanically grinding and dry sieving. Agglomeration prevented 
the use of sedimentation. The sixth fraction was prepared by air milling some of the $-100+200$ fraction in a Trost jetmill at 60 psi air pressure.

Table 2 gives the screen sizes used with the actual particle size determination of the powder before and after pressing. These determinations. were made using both a binocular microscope and an electron microscope. There was excellent agreement between the powder and the pressed pellets indicating that there had not been any significant grain growth or crushing during pressing. The wide ranges in particle size and the similarity in the 2 lower fractions (samples 44 and 45 ) were inherent in the fractionating process and are not an indication of uncertainty in the determination even though the grain boundaries were quite hard to see. The pressed samples were studied both as-pressed and after an alcohol-water etch for various lengths of time, usually starting with a few seconds exposure to the etchant.

The samples were fabricated by pressing at room temperature in a steel die at 15,000 psi for 5 minutes with the resultant densities shown in Table 2. Considerable effort had been expended on hot pressing samples in the vicinity of $400^{\circ} \mathrm{C}$ and 2000 psi. However, the resultant samples did not have sufficient mechanical strength to be usable and the cold pressing at higher pressures was used. The samples in both cases were opaque white.

Some effort was also expended in trying to fabricate polycrystalline samples with very good grain boundaries. The work of Cabarie 4 showed that these transparent samples, while still exhibiting preferential grain boundary diffusion of the anion, did not show enhancement of the anion diffusion coefficient. The procedure followed was to heat the powder to approximately $500^{\circ} \mathrm{C}$ slowly in air, maintain it for 1 to 50 hours with several quick grindings interjected, load it in the steel die which itself was at $\sim 100^{\circ} \mathrm{C}$, and to press immediately. The resultant samples were still opaque unless the pressure was raised to $60-70,000$ psi 
(Cabańe had obtained transparent crystals at $1 / 3$ this pressure). Since the "undried" powder gave similar results, it was concluded that the water had not been completely removed and that a more elaborate preparation procedure was necessary. Several attempts to vacuum hot press at low pressures were also unsuccessful.

Table 2. Particle Sizes and Densities of Polycrystalline Samples

\begin{tabular}{|c|c|c|c|c|c|}
\hline \multirow{2}{*}{ Sample } & \multicolumn{2}{|c|}{ Screen } & \multirow{2}{*}{$\begin{array}{c}\begin{array}{c}\text { Measured } \\
\text { particle size }\end{array} \\
(\mu)\end{array}$} & \multicolumn{2}{|c|}{ Density } \\
\hline & size & range $(\mu)$ & & $\mathrm{gm} / \mathrm{cc}$ & $\%$ theoretical \\
\hline 42 & +100 & $>149$ & $400 \pm 200$ & 2.121 & 97.9 \\
\hline 41 & $-100+200$ & $74-149$ & $\sim 75-150$ & 2.117 & 97.7 \\
\hline 43 & $-200+325$ & $44-74$ & $\sim w /$ some 20 & 2. 103 . & 97.1 \\
\hline 45 & $-325+400$ & $38-44$ & generally 38 & 2.096 & 96.8 \\
\hline 44 & -400 & $<38$ & $20-30$ & 2. 080 & 96.0 \\
\hline 40 & jet milled & & 2 or less & 2.072 & 95.7 \\
\hline $\begin{array}{l}\text { single } \\
\text { crystal }\end{array}$ & & & & 2.166 & 100.0 \\
\hline
\end{tabular}

3. Electrodes. The following electrode materials were tried as explained:

a. DuPont 4887 silver paste, lot 19837, air dried, slowly heated to $400^{\circ} \mathrm{C}$ in air, cooled to room temperature;

b. gold, evaporated on the cold sample;

c. platinum, evaporated on the cold sample;

d. DuPont 7447 liquid bright platinum, air dired at $100^{\circ} \mathrm{C}$, fired at $500^{\circ} \mathrm{C}$ in air for 5 minutes. 
Since, no variation was found in the measured conductivity as a function of electrode material, the bright platinum was used because it was the easiest to work with.

After wiping the surfaces with absolute alcohol the bright platinum was painted on the face. The resistivity of the coating was checked after firing. The sample was repainted until the electrode's surface resistivity was less than $15 \mathrm{ohms} / \mathrm{cm}$ (generally 2 coats). After both faces were coated, a gap to provide a guard ring was scratched in one face. The surface impedances (high to guard, low to guard) and the impedance from high to low were measured to make sure there were no low resistance paths or shorts.

4. $\mathrm{t} / \mathrm{A}$ Determination. Immediately after electroding, a slide of known magnification was taken of the high electrode using a Kodak Polaroid MP-3 Land Camera. The sample's thickness was measured (prior to electroding) to 3 significant figures using a micrometer. Generally at a later time, after the sample was already in the sample chamber, the area of the high electrode was determined. The picture of the sample's electrode was projected on a piece of graph paper so that a known magnification was obtained. The outline was traced and the area within the outline determined using a planimeter. Knowing the magnification, the high electroded area was calculated and was combined with the measured thickness to obtain the t/A ratio.

Since the data for a sample with several different electrodes all fell on the same curve within the experimental scatter, it was felt that the error in the $t / A$ determination was well within this scatter. However, even though this was true for single crystal samples and samples of a given particle size, it is not proof that a systematic error in the effective area could not occur as a function of particle size. 
B. Sample holder

Figures 1 and 2 are pictures of the sample holder, with Figure 2 showing the sample in place and the ceramic shield in the raised position. Figure 3 is a simplified drawing of the holdex used to point out several features of the holder's construction as outlined below.

1. The sample's electrodes contact only platinum $+10 \%$ rhodium foil.

2. The high and low measurement leads are the platinum legs of the two platinum-platinum $+10 \%$ rhodium thermocouples. The reported temperatures were the average of the two readings. Within the experimental scatter no difference in conductivity was determined upon heating or cooling unless the rate was quite rapid, indicating that equilibrium data were being obtained.

3. The high and guard contacts are spring loaded so as to maintain good electrical contact with the sample at all times.

4. The high and low leads are completely guarded so that any leakage currents were to guard and therefore across the transformer or detector for a.c. measurements (see Figure 10) and across the $\mathrm{X}_{1}$ amplifier for the d.c. measurements (see Figures 8 and 9).

5. The ceramic parts were all fabricated from high temperature, high purity, polycrystalline alumina. Any leakage paths across these parts would be orders of magnitude greater in resistance than the sample's resistance at all temperatures.

6. The entire shield assembly can be raised as a unit for easy access of the sample in the sample holder.

After placing the sample in the sample holder the continuity from the holder's leads to the sample's electrodes was checked to insure good contact to the sample. Also the impedances - high to guard, low and ground, low to guard and ground and guard to ground - were all measured at the sample holder's leads to determine if there were any low impedances paths or shorts in either the holder or the sample. 


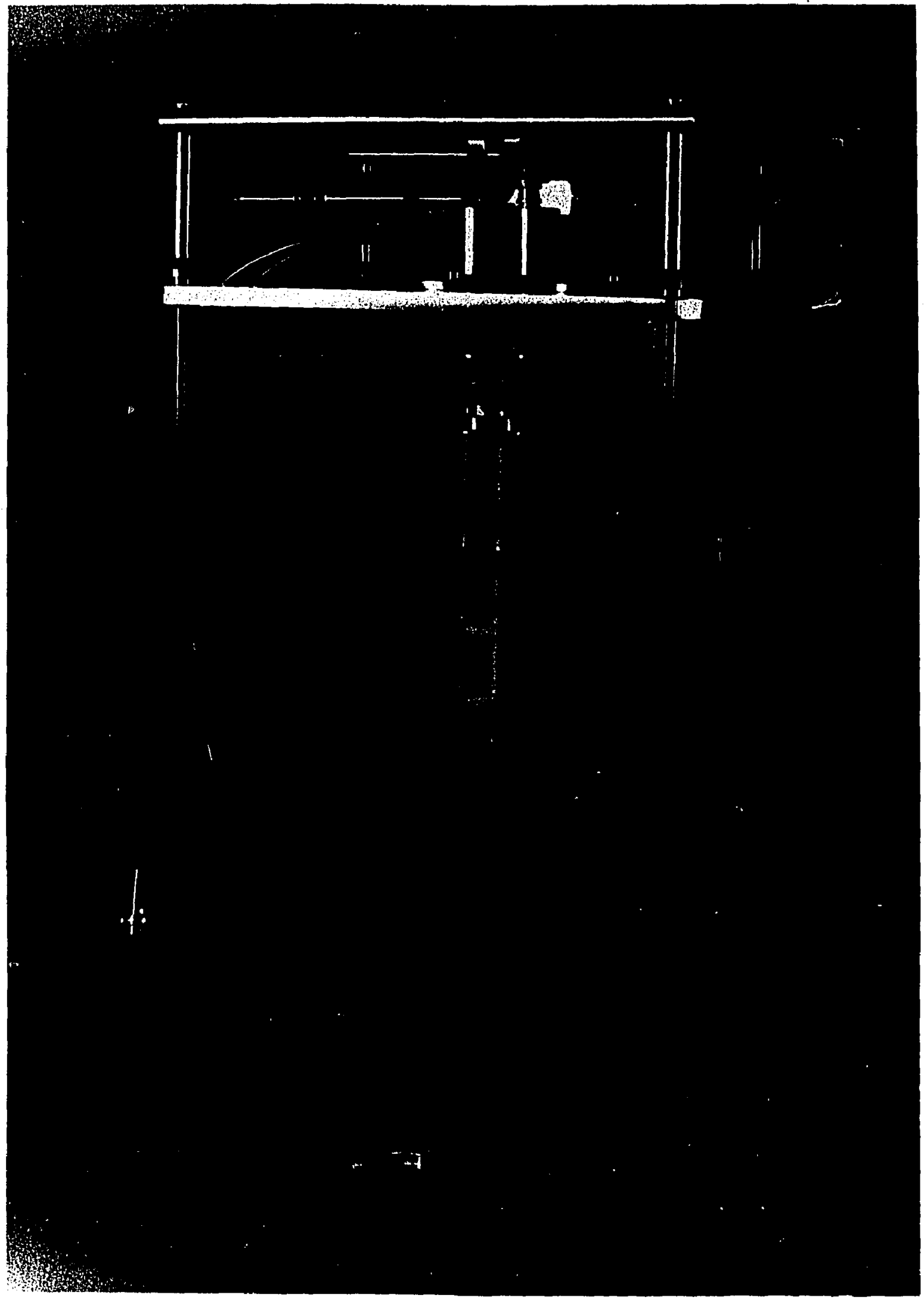

Figure 1. Sample Holder 


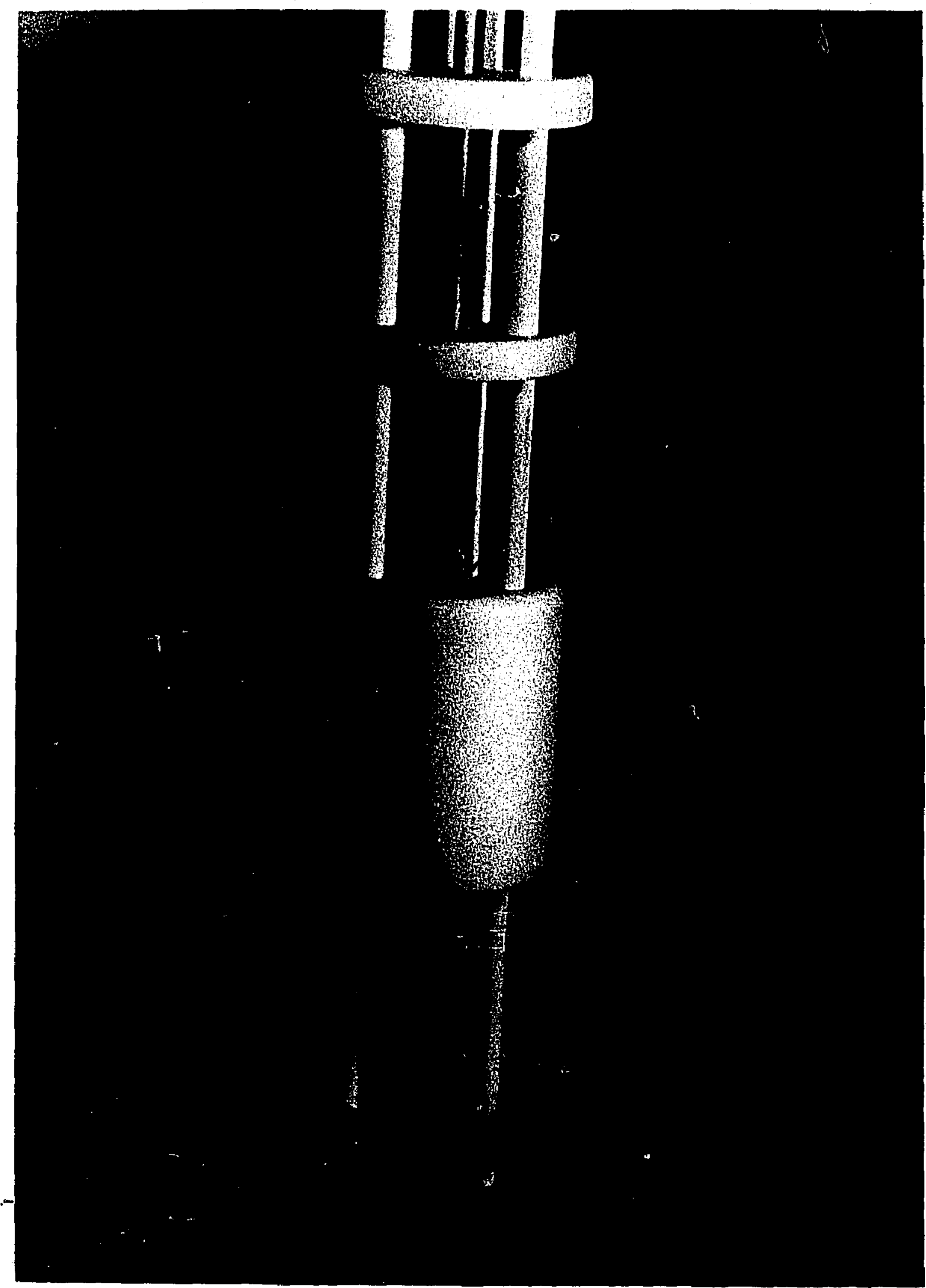

Figure 2. Close-Up of Sample in Sample Holder 


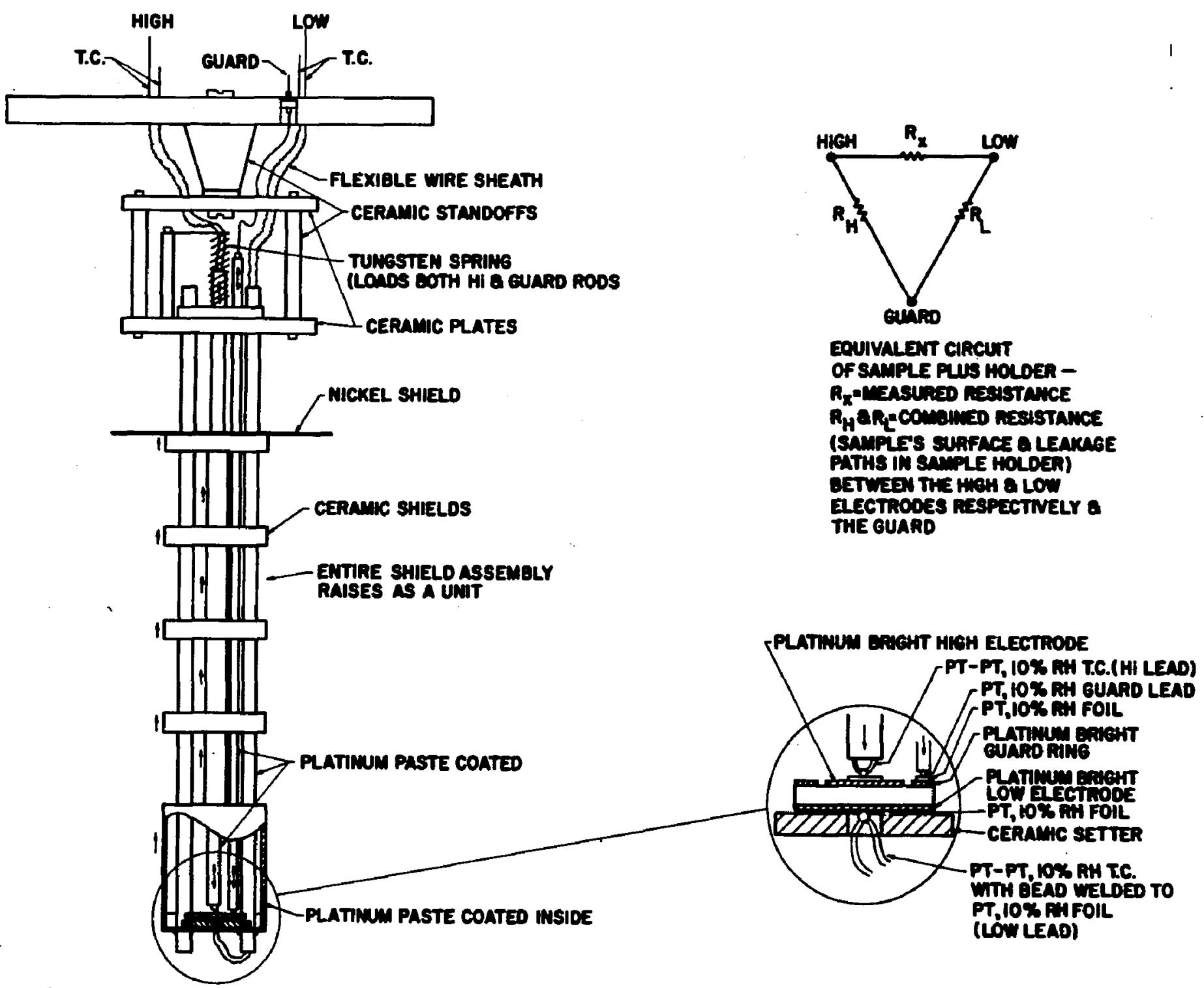

Figure 3. Simplified Drawing of Sample Holder 
C. Sample environment

Figure 4 is a schematic representation of the sample holder in place. in the furnace with the basic auxillary equipment used to control the sample chamber environment.

The sample holder was lowered into the sample chamber while the furnace was maintained at a temperature in the vicinity of $300^{\circ} \mathrm{C}$. Temperature equilibrium was obtained, the sample chamber pumped out to $10^{-4}$ Torr, and the temperature raised slowly to about $525^{\circ} \mathrm{C}$. This initial heating to $\sim 525^{\circ} \mathrm{C}$ was found to be necessary in order to get reproducible data at lower temperatures. Although no pressure dependence of the conductivity was seen, the sample chamber was valved off after this heat treatment and the remainder of the test conducted in a static vacuum of approximately $10^{-3}$ Torr total pressure.

A high temperature, impervious alumina tube separates the sample chamber environment from the furnace chamber vacuum. The vacuum for the resistance-type tungsten wound furnace is maintained below $5 \times$ $10^{-5}$ Torr. The water cooled collets at both ends of the ceramic tube serve to both hold the tube in place and to cool the o-ring seals. A sliding fit is maintained at the top o-ring to allow for the expansion and contraction of the tube during heating and cooling. Approximately $5 \mathrm{KW}$ at 110 volts is necessary to maintain the sample chamber at $1800^{\circ} \mathrm{C}$. The construction and shielding of the furnace helped to minimize the possibility of ionization affecting the heating element and also to eliminate interference or pickup of furnace noise at the sample. Figure 5 is a picture of the assembled furnace with the alumina tube and cullets in place.

D. Measuring equipment

1. General. The block diagram of the overall arrangement of the measuring equipment is given in Figure 6. The switch shown makes 


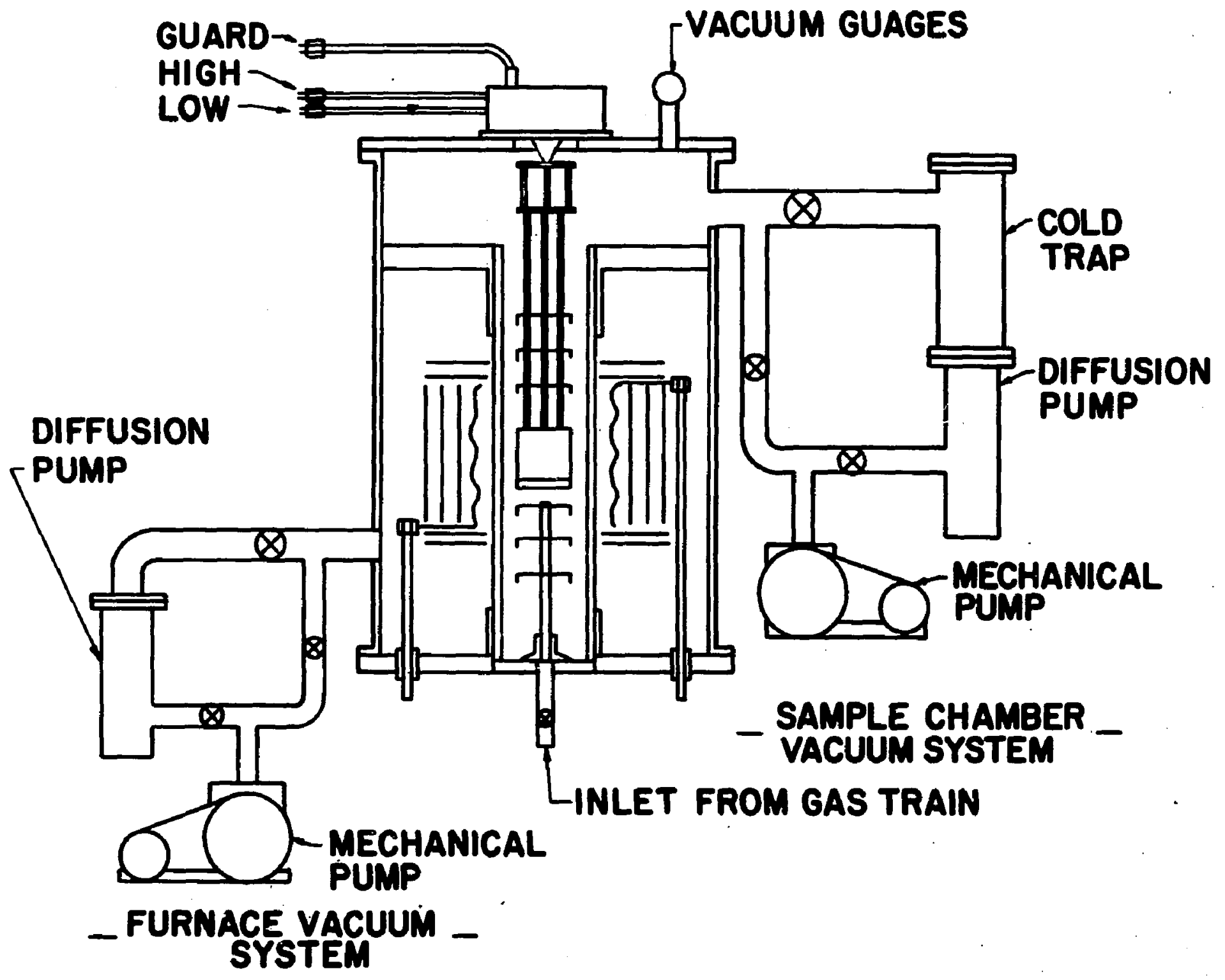

Figure 4. Schematic of Environment Control Equipment 


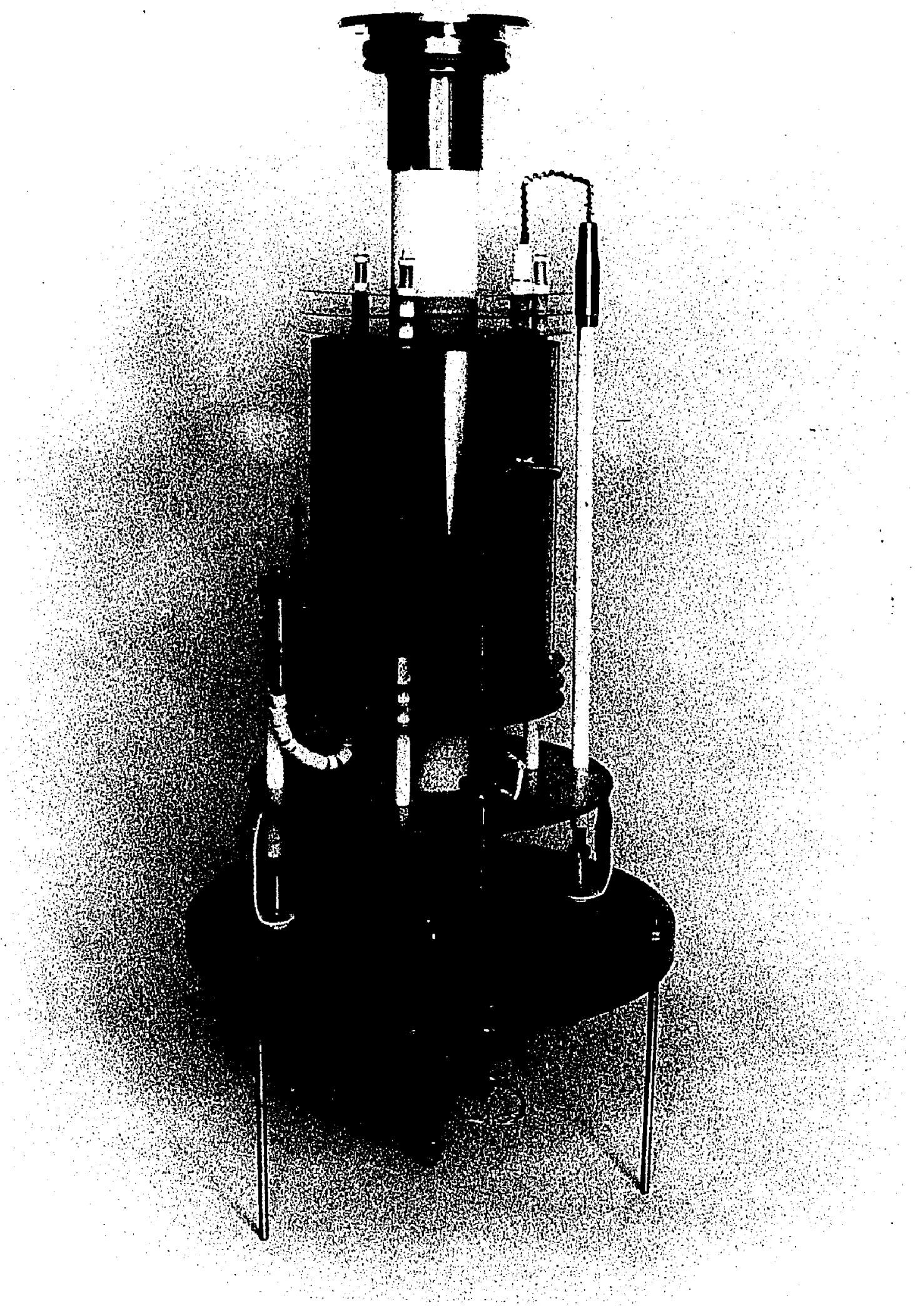

Figure 5. Furnace Assembly 


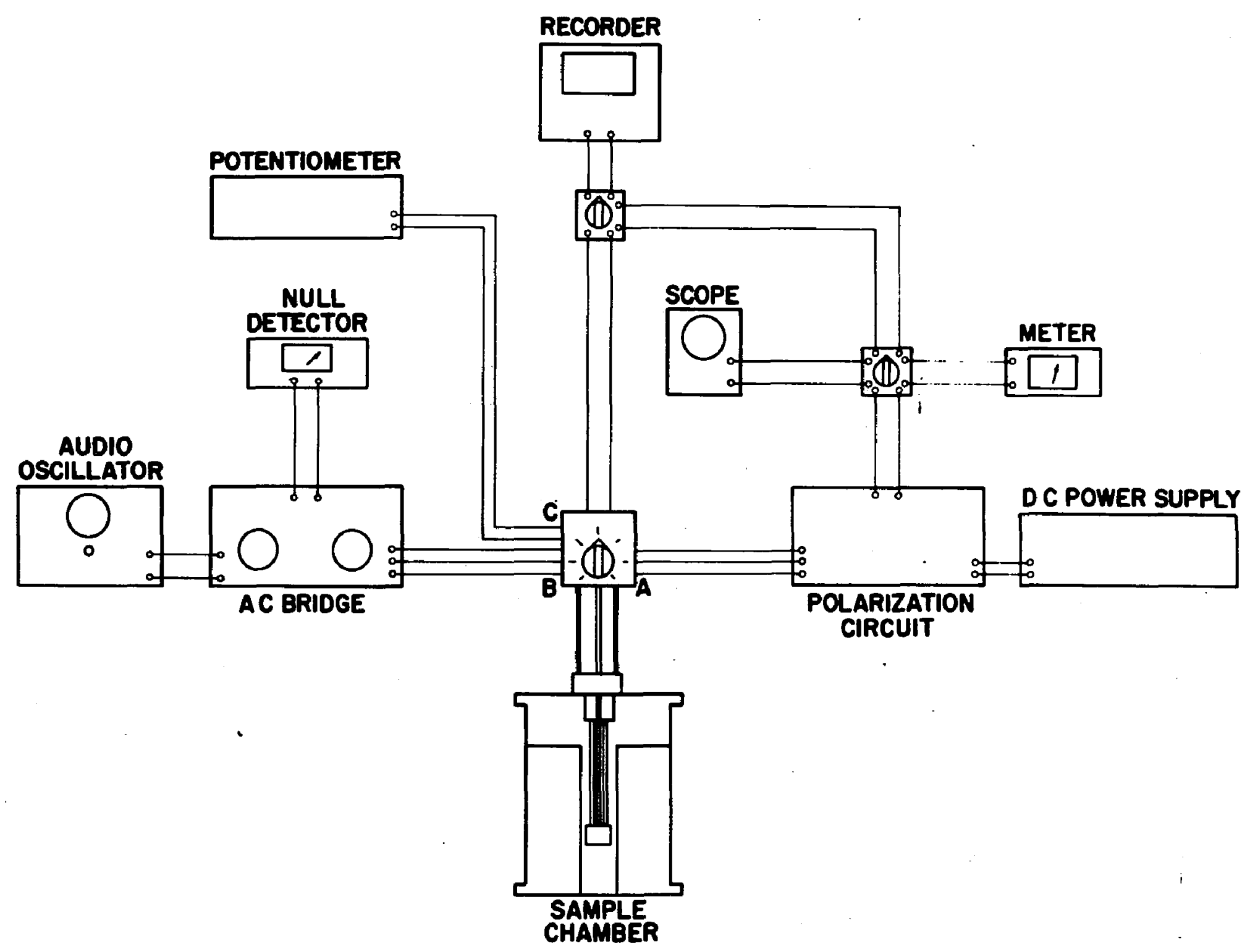

Figure 6. Block Diagram of Measuring Equipment. 
any one of the 3 indicated measurements possible at a given time without physically switching the leads. That is, in position "A", d. c, polarization measurements or in position "B", a. c. bridge measurements or in position "C", a readout of the thermocouple's emf on either the potentiometer or the recorder can be made. All leads are electrostatically shielded and the measurement leads are completely guarded.

2. d.c. Conductivity. The wiring diagram of the polarization equipment is shown in Figure $7^{30}$ Figures 8 and 9 are the schematic representation of this circuit for the constant voltage and the constant current modes, respectively.

In Figures 8 and 9 the sample is represented by a parallel network consisting of resistances $R_{N B}$ and $R_{B}$ and capacitance $C$. $R_{N B}$ is the resistance attributed to the non-blocked carrier, that is the sodium ion vacancy in this work, but usually the electron. $R_{B}$ and $C$ represent the blocked carrier. These two, in series, form an RC circuit which has a time constant determined by the magnitudes of $R_{B}$ and $C$. In the sample, the limited movement of the blocked ion, determined by its mobility, is analogous to $R_{B}$, while the build up of charge at the electrode, tending to create a back emf, is anologous to the charging of $C$. Initially the total conductance of the sample is determined by the flow of current through both legs (the quantity through each being proportional to its transference number), but at the steady state condition the current flow will be much greater in the non-blocked leg, i.e., $\sigma_{0}$ is the sum of the conductivities of the two carriers and $\sigma_{\infty}$ is the conductivity of the non-blocked carrier.

In the constant voltage mode (Figure 8) the change in current passing through $R_{s}$ is measured as a function of time. In order that the voltage remains constant within $1 \%$ as the resistance of the sample changes during polarization, $R_{S}$ is set at least 2 orders of magnitude less than the total resistance of the sample. The diagram of the change in 


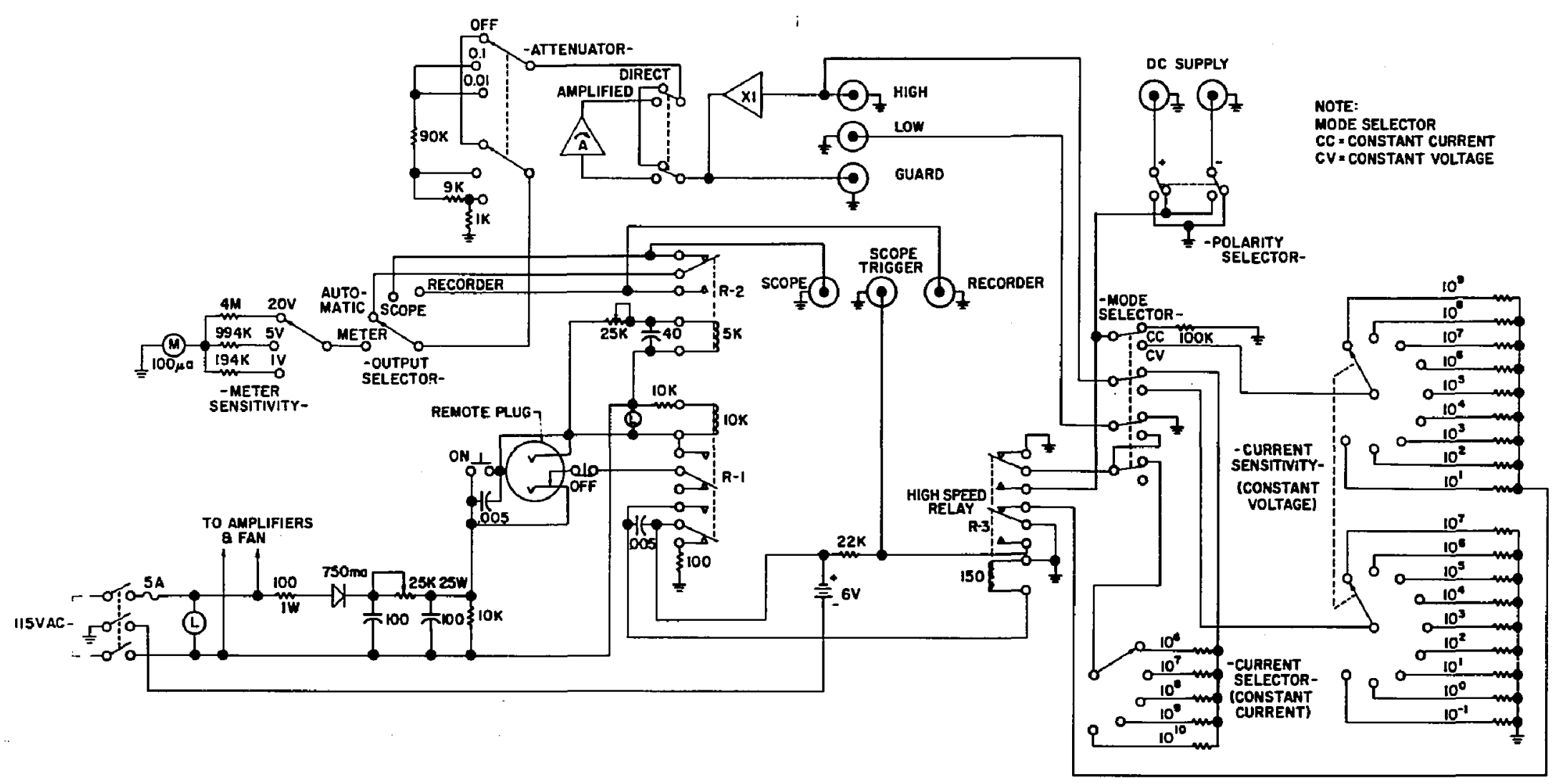

Figure 7. Wiring Diagram of Polarization Equipment 


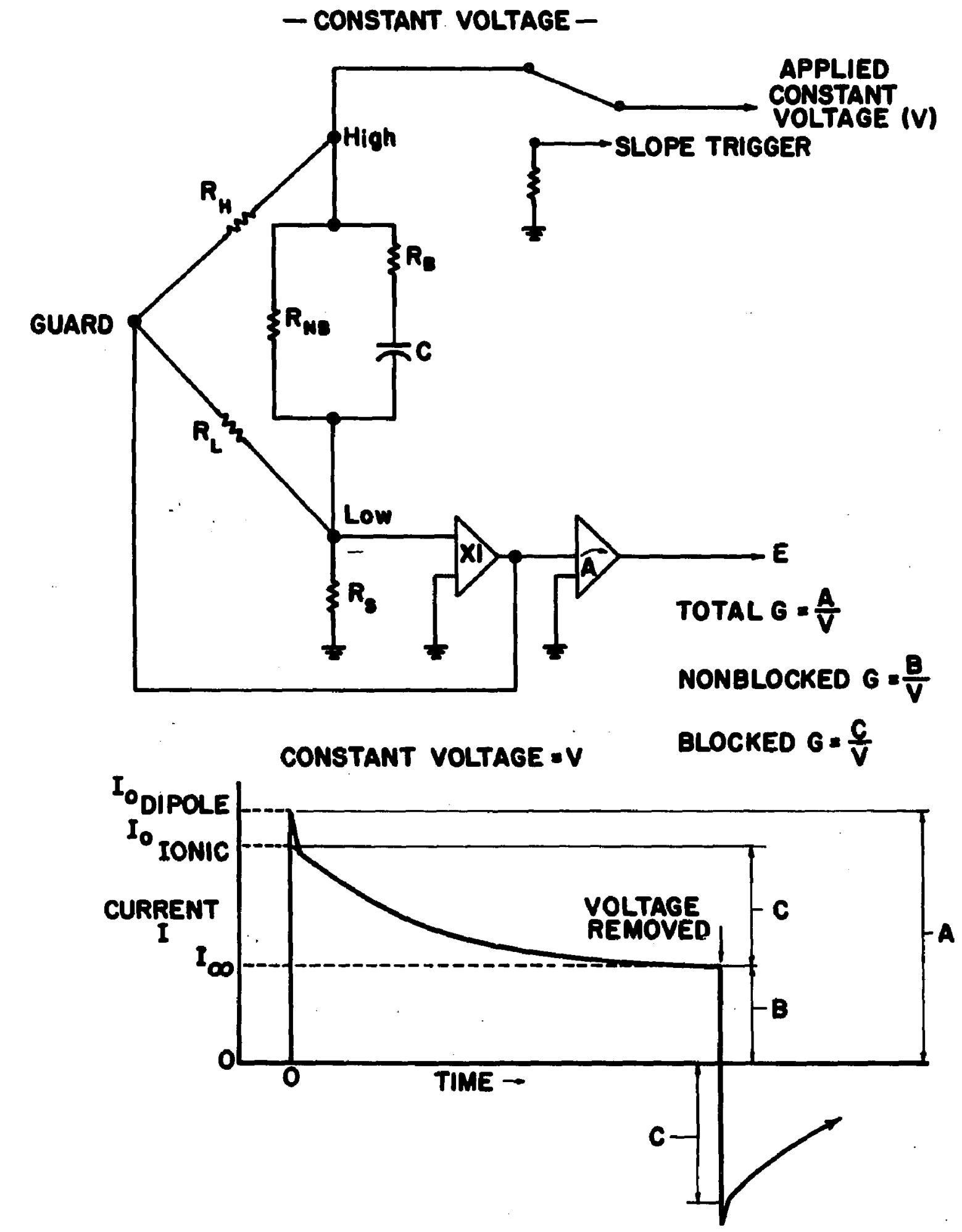

Figure 8. Schematic of Polarization Circuit - Constant Voltage 


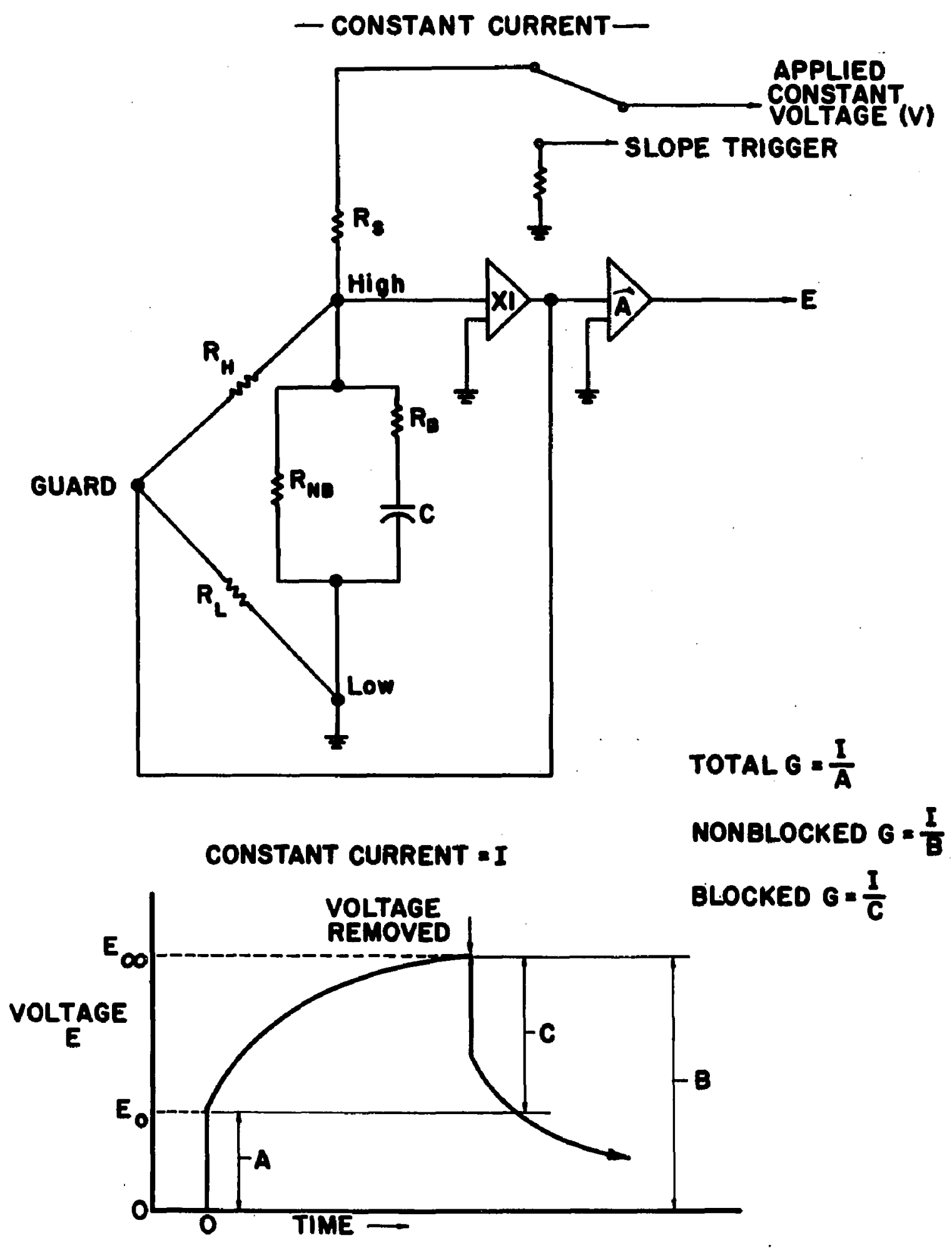

Figure 9. Schematic of Polarization Circuit - Constant Current 
current as a function of time is for a sample which has three conduc tion mechanisms, two blocked and one unblocked. The two blocked mechanisms are distinguished from one another by their different time constants. As shown, the first one seen would be a very fast mechanism such as a dipole rotation and the entire polarization of the dipole would be completed in a fraction of a second. The second polarization process shown would have a longer time constant and could quite pos sibly be the build up of charge at an electrode or an interface due to the movement of an ion to this block and not beyond it. The elimination of this particular ionic species from the conduction process means a decrease in the current passed through the material and hence a decrease in its conductivity. The third conduction mechanism represented is for a charge carrier which can pass from the sample to the external cir cuit. This carrier is normally the electron but as in the case of $\mathrm{NaCl}$ it may well be an ionic carrier which is not blocked by the electrodes ( $\mathrm{Na}$ ion vacancy).

The time constant of the polarization process determines the read out equipment necessary. The dipole type mechanism requires the use of a fast risetime scope while an ionic mechanism may take several minutes to several hours to complete, depending upon the material and the temperature, and may be recorded on a normal recorder. The scope and recorder used in this work were the Tektronix Type 555 Oscilloscope with Type $H$ plug in unit (23 $\mathrm{n}$ sec risetime) and a Leeds and Northrup Type "H" Azar recorder.

Since the measurement made was of the change in voltage across a standard resistor, the current through the sample was

$$
I=\frac{E}{a R_{s}} \text { (amps), }
$$

where $\quad E=$ the measured potential in volts at a given time,

$a=$ any amplification of $\mathrm{E}$,

$R_{s}=$ the standard series resistance in ohms. 
It follows then that the conductance would be

$$
G=\frac{I}{V}=\frac{E}{a R_{s} V}(\text { ohms })^{-1},
$$

where $\mathrm{V}$ is the applied constant voltage, and the conductivity is given by

$$
\sigma=\frac{G t}{A}=\frac{E t}{a R_{s} V A}\left(0 h \mathrm{~ms}^{-1} \mathrm{~cm}^{-1}\right) \text {, }
$$

where $t=$ the sample thickness in $\mathrm{cm}$,

$A=$ the high electrode area in $\mathrm{cm}^{2}$.

In calculating the various conductivities, using $E_{O}$ for $E$ in (7) would give the total conductivity; $\mathrm{E}_{\infty}$ for $\mathrm{E}$ the unblocked conductivity, and the difference in the two, $\sigma_{0}-\sigma_{\infty}$, would be the blocked conductivity. The subscripts $O$ and $\infty$ refer to zero time and steady state values, respectively.

- In the circuit operation the XI amplifier (Cohu amplifier model 112A) maintains the low and guard points at the same potential. Due to its high d. c. impedance (1010 ohms) it does not affect the current passing through $R_{S}$. Since $R_{L}$ appears as an infinite resistance with the guard and low points at the same potential, the voltage drop measured across $R_{s}$ will be a function only of the current passed through the sample.

Similar comments can be made for the constant current schematic and voltage vs. time plot shown in Figure 9. Here, of course, the change in voltage across the sample as a function of time for a con stant current is measured. The $\mathrm{Xl}$ amplifier maintains the high and the guard points at the same potential to again minimize the effect of surface currents. $R_{s}$ is at least 2 orders of magnitude greater than the samples total resistance.

The calculation of the conductivity is

$$
\text { r } \begin{aligned}
\sigma & =\frac{V t}{a E R_{B} A}, \\
\text { since } \quad G & =\frac{I}{a \cdot E} \text { and } I=\frac{V}{R_{S}}=\text { constant. }
\end{aligned}
$$


Here again, substituting $\mathbf{E}_{\mathrm{o}}$ for $\mathrm{E}$ in (8) gives the total conductivity, $\mathrm{E}_{\infty}$ for $\mathrm{E}$ the unblocked, and the blocked conductivity is $\sigma_{0}-\sigma_{\infty}$.

In both modes the current passed through the sample was held at the lowest level consistant with the output of a signal large enough to be detected.

The transference number of either of the two carriers is calculated from the conductivities as follows :

$$
t_{\mathrm{NB}}=\frac{\sigma_{\infty}}{\sigma_{0}} \text { and } t_{B}=\frac{\sigma_{0}-\sigma_{\infty}}{\sigma_{0}}
$$

where $t_{N B}$ and $t_{B}$ are the non-blocked and blocked transference numbers, respectively.

3. a.c. Conductivity. The a.c. conductivity measurements were made using a Wayne Kerr B 221 Universal Bridge. This is a trans former ratio arm bridge with an internal oscillator adjusted to $1592 \mathrm{c} / \mathrm{s}$ $\left(\omega=10^{4}\right.$ radians $)$, but also with the capability of using an external audio oscillator and suitable detector. When the conductivity was measured as a function of frequency a Hewlett-Packard 241A pushbotton oscillator was used as the signal source and a General Radio 1232-A tuned amplifier and null detector as the detector.

Figure 10 shows a simplified circuit arrangement for the bridge and sample. The sample's resistance and the leakage resistances $\left(R_{X}, R_{H}\right.$ and $R_{L}$ ) are shown in place. The standards of conductance and capacitance are represented by $R_{s}$. The outstanding advantage of the transformer bridge in making a three terminal, guarded, measurement is the elimination of the tedious balancing procedure previously necessary when using a Schering bridge and guard circuit combination. In the transformer bridge the stray or leakage impedances are shunted across the source or detector. This makes it possible to measure materials which are being held in a sample holder without incurring an error due to these other paths. 

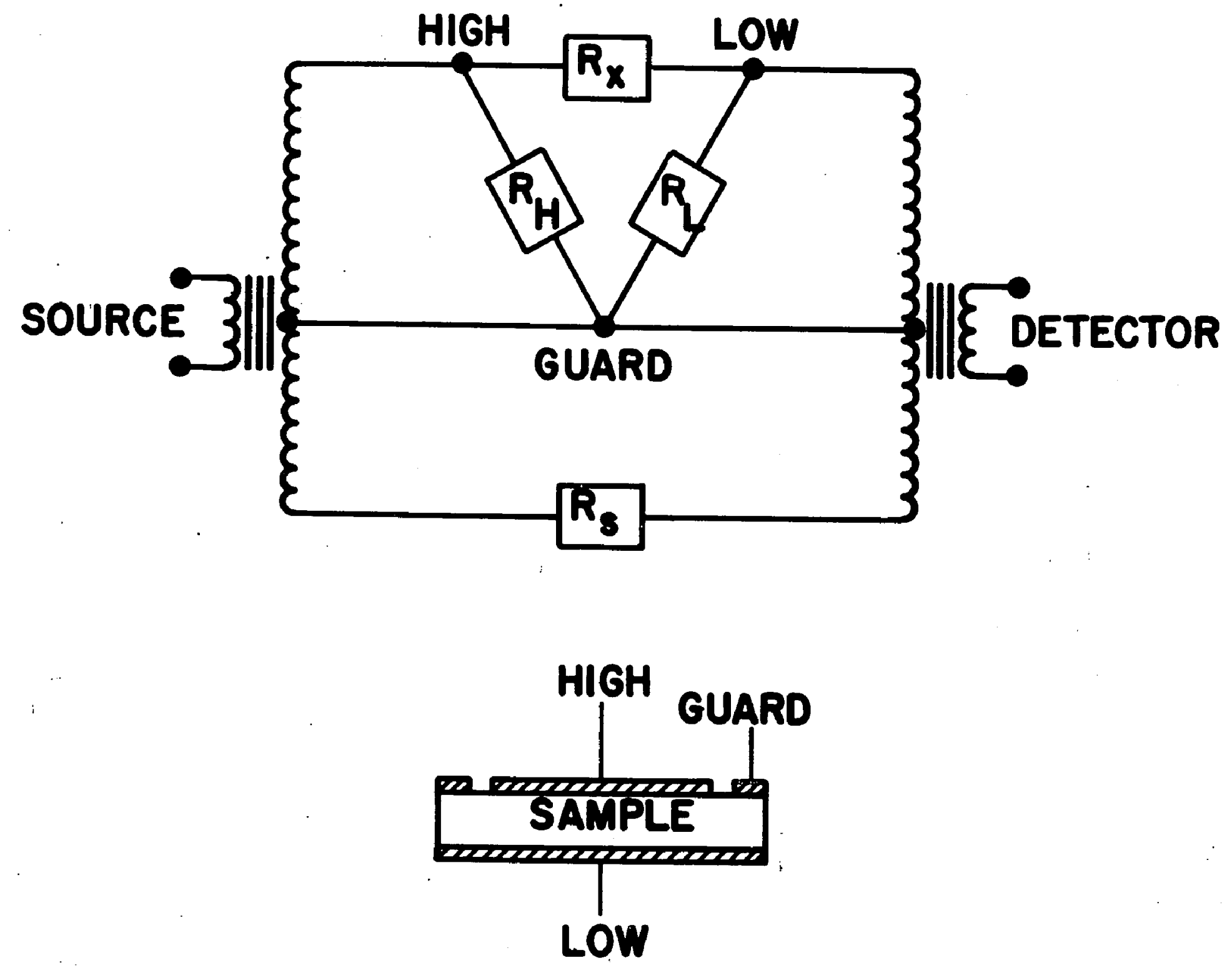

Figure 10. A. C. Circuit Schematic 
4. Readout. The previously mentioned recorder was used to both monitor temperature, sample's or furnace's, and to record the long time polarization data. Temperature measurement was by a Leeds and Northrup 8686 millivolt potentiometer. The fast time polarization data was recorded using a Tektronix C-l2R camera. Regardless of how the data were obtained - a.c., d. c., recorder or oscilloscope - the results were consistent and quite reproducible. The scatter of the data was quite small compared to the changes being observed.

5. Density determination. The density determinations were made using the Archimedes Principle. Ethylene bromide (1, 2 - dibromoethane) was used as the liquid because of its relatively high density and because it wets $\mathrm{NaCl}$ well. The following formula ${ }^{31}$ was used to determine the density versus temperature curve for the liquid:

$$
\begin{aligned}
a_{t} & =\left[d_{s}+10^{-3} a\left(t-t_{s}\right)+10^{-6} \beta\left(t-t_{s}\right)^{2}+10^{-9} \gamma\left(t-t_{s}\right)^{3}\right] \\
& \pm 10^{-4} \Delta(\mathrm{gm} / \mathrm{cc})
\end{aligned}
$$

where $\quad d_{t}=$ the density at temperature $t$

$$
\begin{aligned}
& t=\text { the temperature of interest } \\
& t_{s}=0^{\circ} \mathrm{C} \\
& d_{s}=\text { density at } t_{s}(2.2223 \mathrm{~g} / \mathrm{cc} \text { by extropolation }) \\
& a=-2.090 \\
& \beta=-0.20 \\
& \gamma=0 \\
& \Delta=2 \times 10^{-4}
\end{aligned}
$$

range $0-30^{\circ} \mathrm{C}$, probable error $5 \times 10^{-4}$ or less.

A thermometer graduated in $0.10^{\circ} \mathrm{C}$ divisions was used to determine the liquid temperature and a constant load balance to determine the mass of the sample. The difference of the sample's weight in air and in the liquid was divided by the density of the liquid to obtain the volume of the sample. Its mass in air divided by this volume equals the sample's density. 
Several single crystal pieces were measured and the results agreed with those of Cabarie to within $0.1 \%$.

6. Additive coloring. Several pieces of the Harshaw boule were additively colored by exposing them to a sodium vapor atmosphere. In each case a stainless steel bomb was made similar to the one shown in Figure 11. The silver soldering of the top had to be done after the sample in its wire suspension was placed in the bomb. After soldering on the top, several pieces of sodium metal were dropped in through the pumping tube. The bomb was then pumped out and held at about $10^{-3}$ Torr while the tube was sealed and then soldered. The entire unit was placed in an oven at a temperature in the vicinity of $550^{\circ} \mathrm{C}$ for a period of from 16 to 72 hours. Upon removal from the oven the bomb was water quenched to room temperature. 


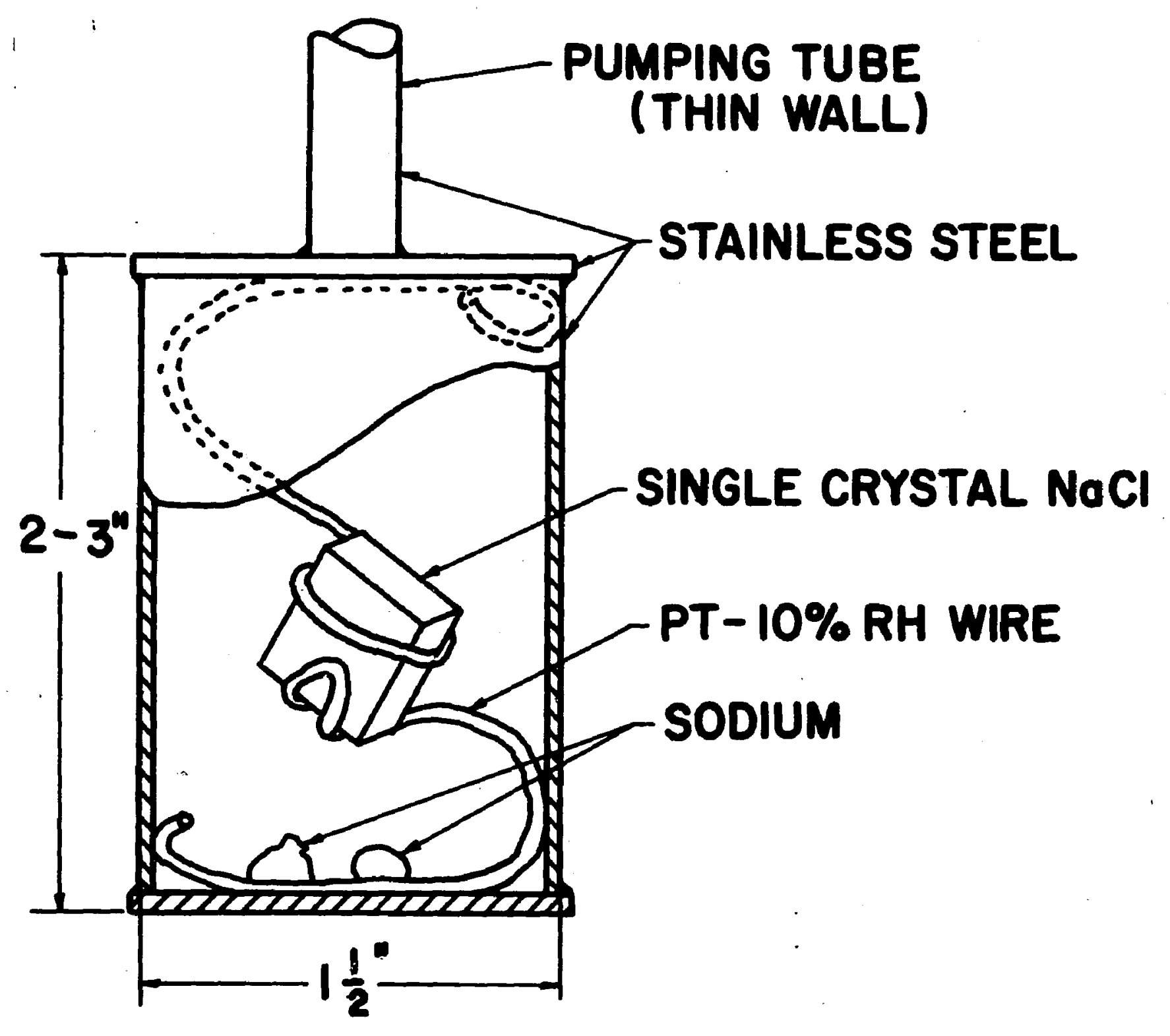

Figure 11. Bomb Used to Additively Color $\mathrm{NaCl}$ 


\section{RESULTS AND DISCUSSION}

1. Single crystal, as-received. In order to establish a base curve for this work, the conductivity of the as-received single crystal from the Harshaw Chemical Company was measured as a function of temperature. Figure 12 is a plot of both the a.c. and the d.c. conductivities as a function of reciprocal temperature. The activation energies shown for this sample and for all other samples reported were calculated assuming the curves to obey equations of the form ${ }^{8}$

$$
\begin{aligned}
& \sigma=n e \mu=\left(\frac{4 e^{2} a^{2} \nu_{0}}{k T}\right) n_{i} \exp \left(-\epsilon_{m} / k T\right) \text { for the extrinsic } \\
& \text { region and }=\left(\frac{4 e^{2} a^{2} \nu_{0}}{k T}\right) N \exp \left[-\left(\epsilon_{m}+\epsilon_{f} / 2\right) / k T\right] \text {, for the } \\
& \text { intrinsic region, }
\end{aligned}
$$




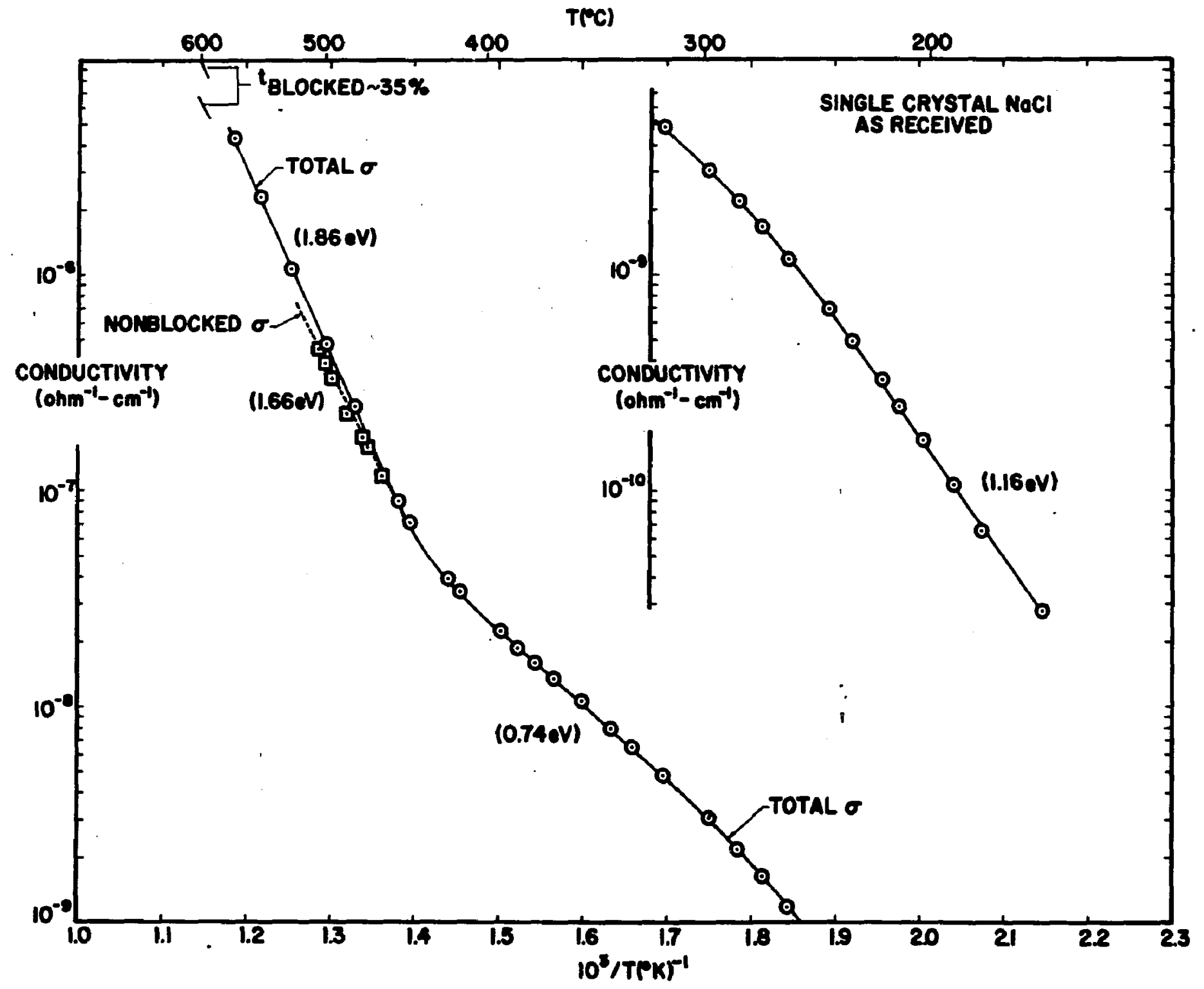

Figure 12. Conductivity versus Reciprocal Temperature for "As-Received" Single Crystal Sample 
Both equations reduce to the form

$$
\sigma T=C \exp (-\epsilon / k T)
$$

and the slope of the logarithm of $(\sigma \mathrm{T})$ versus reciprocal temperature diagram will be the activation energy. The equation used in solving for the activation energies is

$$
\epsilon=\frac{k \ln \frac{\sigma_{1}}{\sigma_{2}}+k \ln \frac{T_{1}}{T_{2}}}{\frac{1}{T_{2}}-\frac{1}{T_{1}}},
$$

where $\sigma_{1}$ and $\sigma_{2}$ are the conductivities at $T_{1}$ and $T_{2}$, and $T_{1}>T_{2}$. The numerator of this equation is larger by $k \ln \left(T_{1} / T_{2}\right)$ than if the pre exponential is assumed to be temperature independent.

Both the activation energies and the absolute magnitude of the a. c. conductivity are in good agreement with the literature. The $1.86 \mathrm{eV}$, intrinsic activation energy, is in exact agreement, the $0.74 \mathrm{eV}$ falls at the low end of a range of energies reported for the impurity controlled sodium vacancy conductivity, and the $1.16 \mathrm{eV}$ is in good agreement with the "association range" activation energy? The position of the "knee" $\left(10^{3} / \mathrm{T} \sim 1.4^{\circ} \mathrm{K}^{-1}, \sigma_{\mathrm{o}} \sim 6 \times 10^{-8} \mathrm{ohm}^{-1} \mathrm{~cm}^{-1}\right)$ also agrees with the literature for "pure" $\mathrm{NaCl}$ single crystals?

The most interesting feature of the data is the line marked "nonblocked $\sigma^{\prime \prime}$. As noted earlier, at high temperatures $\mathrm{NaCl}$ has a polarization phenomenon operative. ${ }^{28,29}$ Therefore, the a.c. conductivity in this range represents the sum of two contributions - a blocked and a non-blocked conductivity.

There are several possible explanations for the polarization seen at high temperatures. It is possible that it results from a dipole rotation mechanism. This is rejected since the time constant for the high temperature polarization was greater than 10 minutes at temperatures as 
high as $500^{\circ} \mathrm{C}$. For the same reason, an interfacial polarization mechanism, due to the build up of charge at an established boundary, was rejected. The results of Tallan and.Wimmer ${ }^{26}$ for $\mathrm{NaCl}$ which were interpreted on the basis of such a mechanism, showed the dielectric loss peak to be at $\sim 10^{4} \mathrm{c} / \mathrm{s}$ at $400^{\circ} \mathrm{C}$ (the time constant would be $\sim 10^{-4}$ seconds). Another possibility is that the electrodes are not completely reversible to cation movement. If a condition like this existed, where there was a maximum flux of cation which the electrode could pass, a build up of charge carriers would occur at the electrode and the repulsive field thus established would eventually limit the current to an amount which the electrode could pass. A third possible explanation is that there are two ionic charge carriers involved and that one of them is blocked.

Qualitatively the single crystal data looks exactly like the results for $\mathrm{KCl}$ obtained by Allnatt and Jacobs. ${ }^{28}$ As mentioned earlier, they did not identify the carrier responsible for the steady state d. c. conductivity, but they did establish that the a.c. curve $\left(\sigma_{0}\right)$ was the sum of two ionic contributions. ${ }^{28,} 29$ Sutter and Nowick's ${ }^{24}$ results, for temperatures below $200^{\circ} \mathrm{C}$, showed that cation conduction was not blocked in $\mathrm{NaCl}$ at low temperatures. That the same is true at high temperatures is not implied. However, results to be presented for one of the additively colored single crystals (Figure 22 ) indicate that it is true. In addition, the results for the polycrystalline samples (Figure 20) show that the non-blocked and blocked conductivities have the same particle size dependence as reported for sodium and chlorine diffusion, respectively, by Laurent and Bénard. ${ }^{3}$ It therefore seems most reasonable that at high temperatures the steady state current is sodium vacancy movement, and the polarization is due to the blocked current of the chlorine vacancy. 
A few general comments apply to Figure 12, as well as to the other conductivity results to be presented. At temperatures in excess of 500 to $525^{\circ} \mathrm{C}$ the total conductivity decreased as a function of time. This decrease was attributed to the loss of electrodes, since both the intrinsic and extrinsic portions of the curve would decrease by the same in crement of conductivity for a given time at temperatures. Also, samples held for long times above $525^{\circ} \mathrm{C}$ showed considerable electrode deterioration upon removal from the sample holder. Short-time ora.c. points could be taken above $575^{\circ} \mathrm{C}$, as they were for the "as received" sample, if the time at these temperatures was kept very short. In all cases, re-electroding the sample would return the curve to its original position.

It was necessary to preheat most samples to a temperature in the intrinsic range to eliminate what appeared to be a non-equilibrium extrinsic conductivity. The fact that this curve of higher conductivity always seemed to have approximately the same slope, suggests the possibility that the annealing process is removing or reorganizing gross imperfections such as dislocations, as proposed by Barr et al. ${ }^{12}$ for chlorine diffusion in $\mathrm{NaCl}$. However, even though there appears to be a different activation energy, a more reasonable picture might be the presence of a "frozen-in" concentration of cation vacancies ("frozen-in" during the electroding process) since the annealing time seems to be very short (a few minutes as compared to hours in the work of Barr et a1.). The slope and position of this curve with respect to the "true" curve, measured after annealing, seemed to be independent of the fabrication pressure and the particle size.

2. Polycrystalline, non-doped. The conductivity curves for the six grain sizes examined are presented in Figures 13 thru 18. Three curves are shown in each figure. The circles represent the total conductivity at time zero $\left(\sigma_{0}\right)$ and the triangles represent the long time, or 


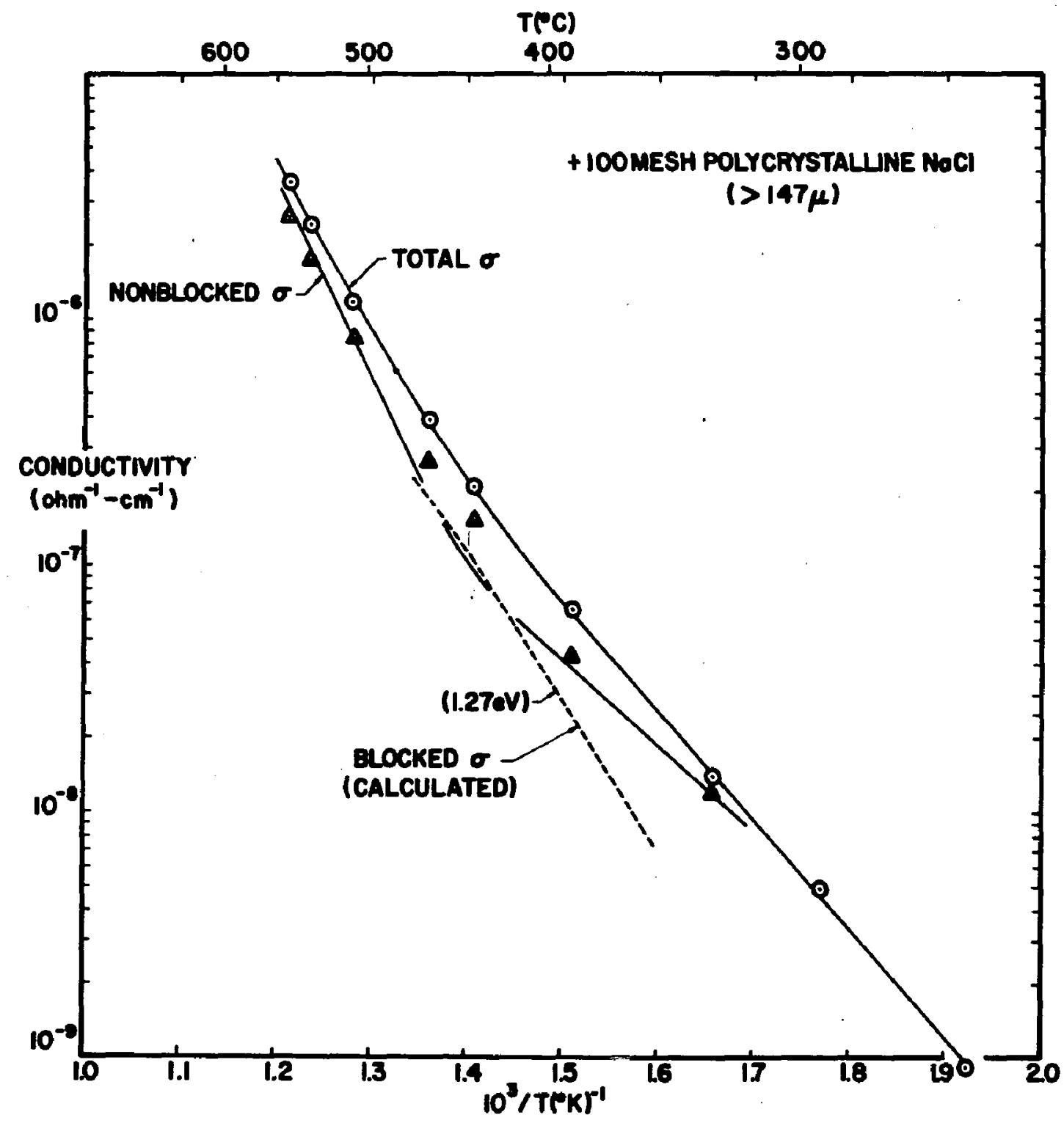

Figure 13. Conductivity versus Reciprocal Temperature for Polycrystalline Sample 


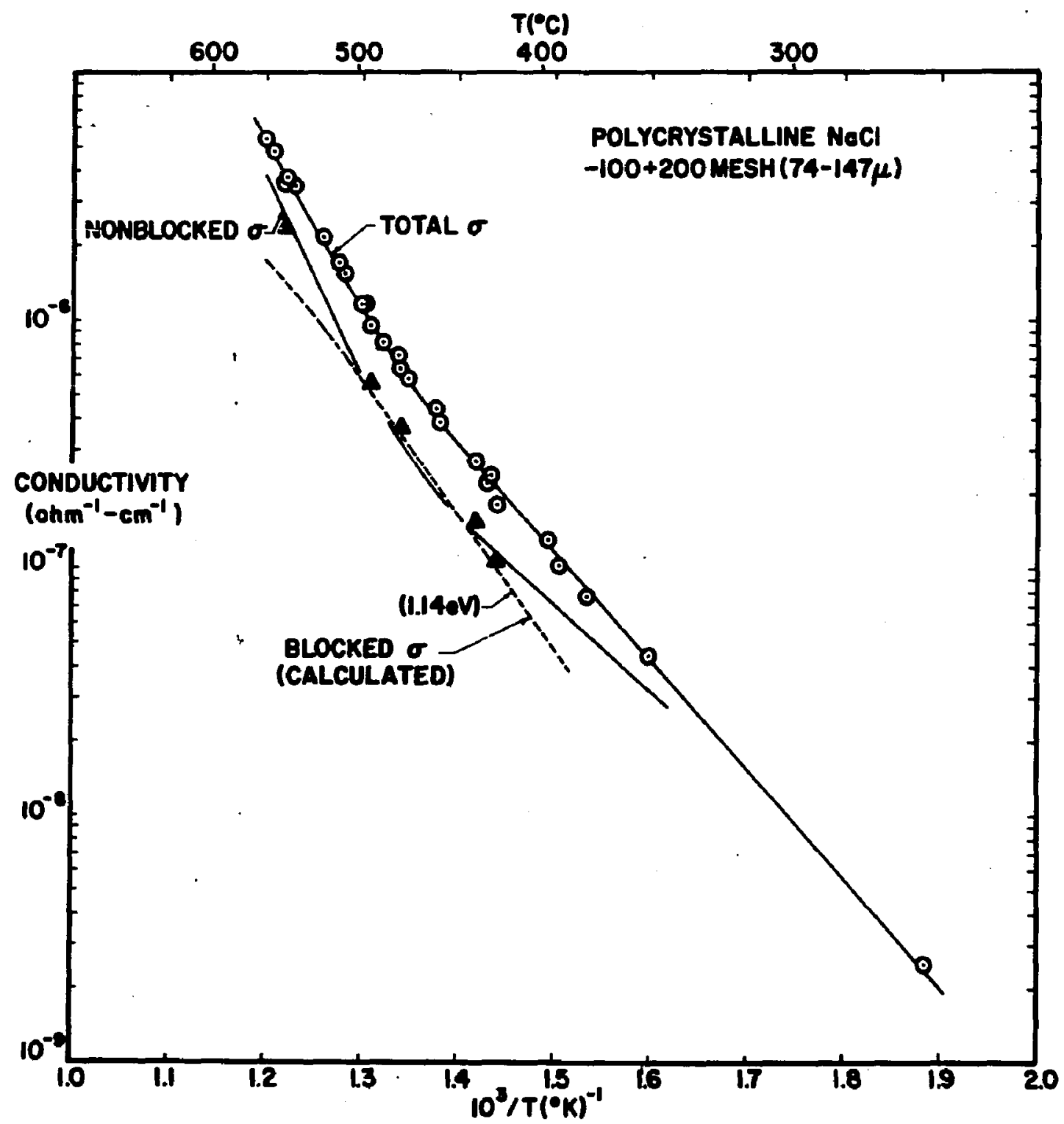

Figure 14. Conductivity versus Reciprocal Temperature for 


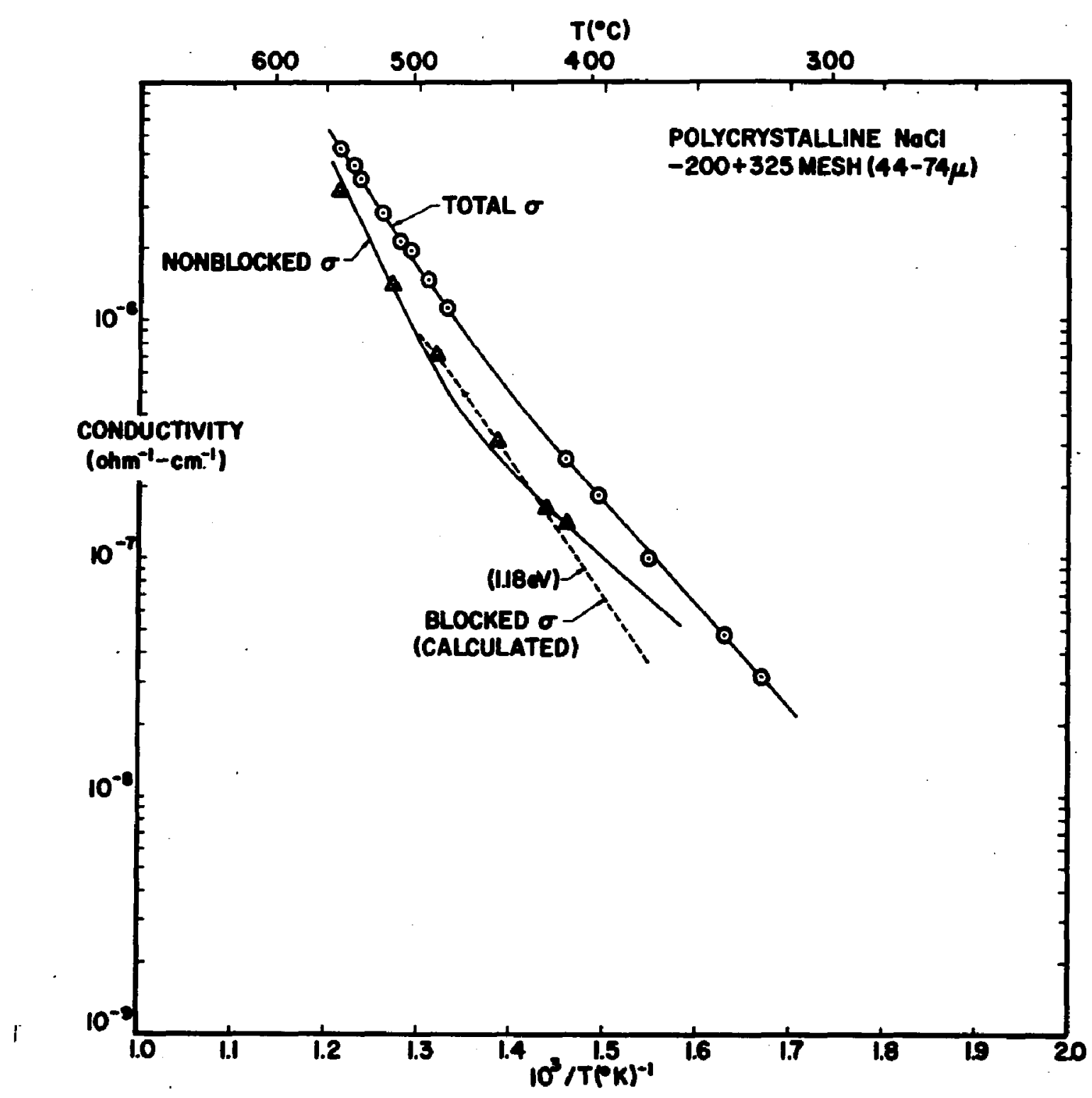

Figure 15. Conductivity versus Reciprocal Temperature for Polycrystalline 


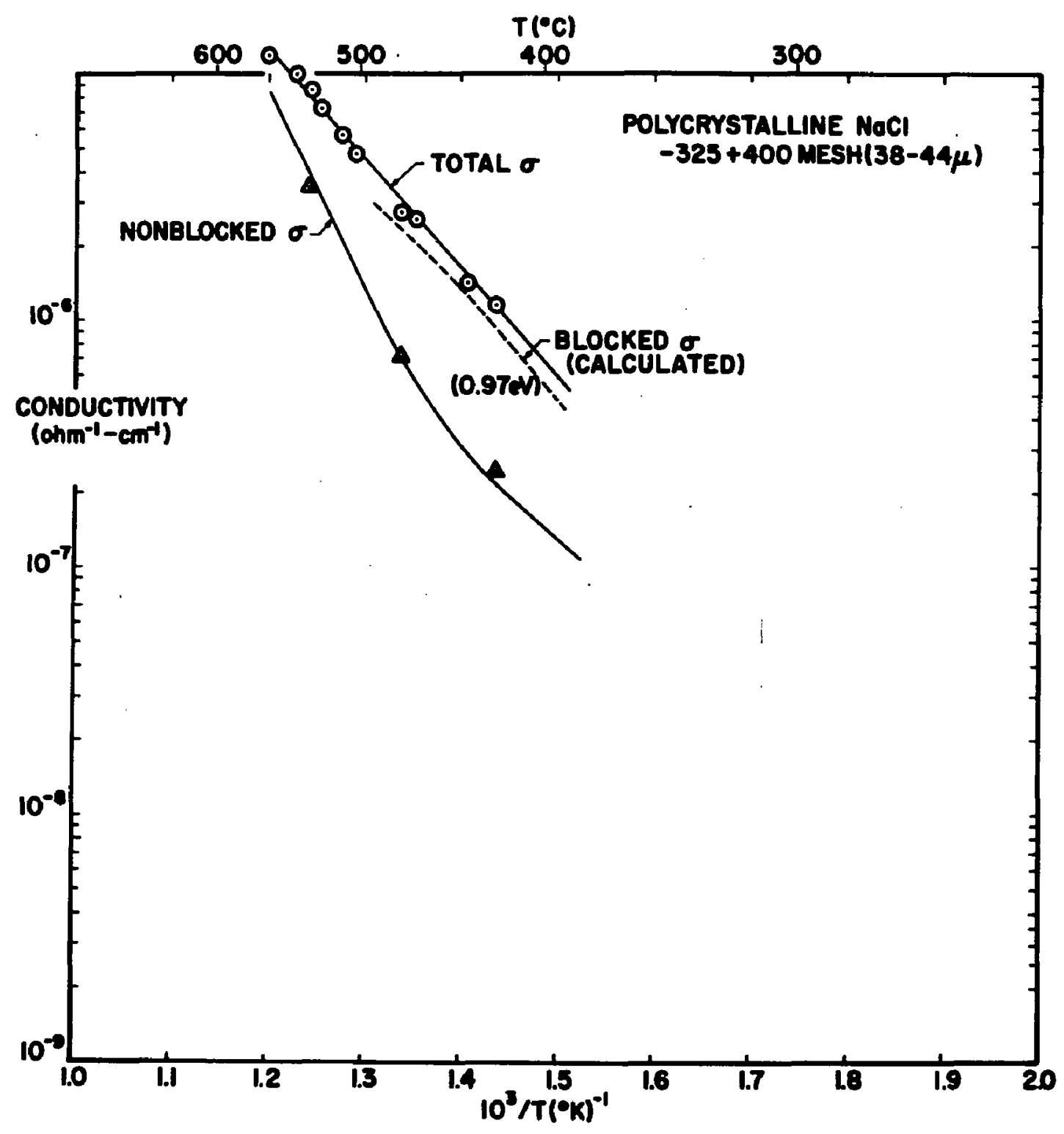

Figure 16. Conductivity versus Reciprocal Temperature for Polycrystalline Sample $-325+400 \mathrm{Mesh}$ 


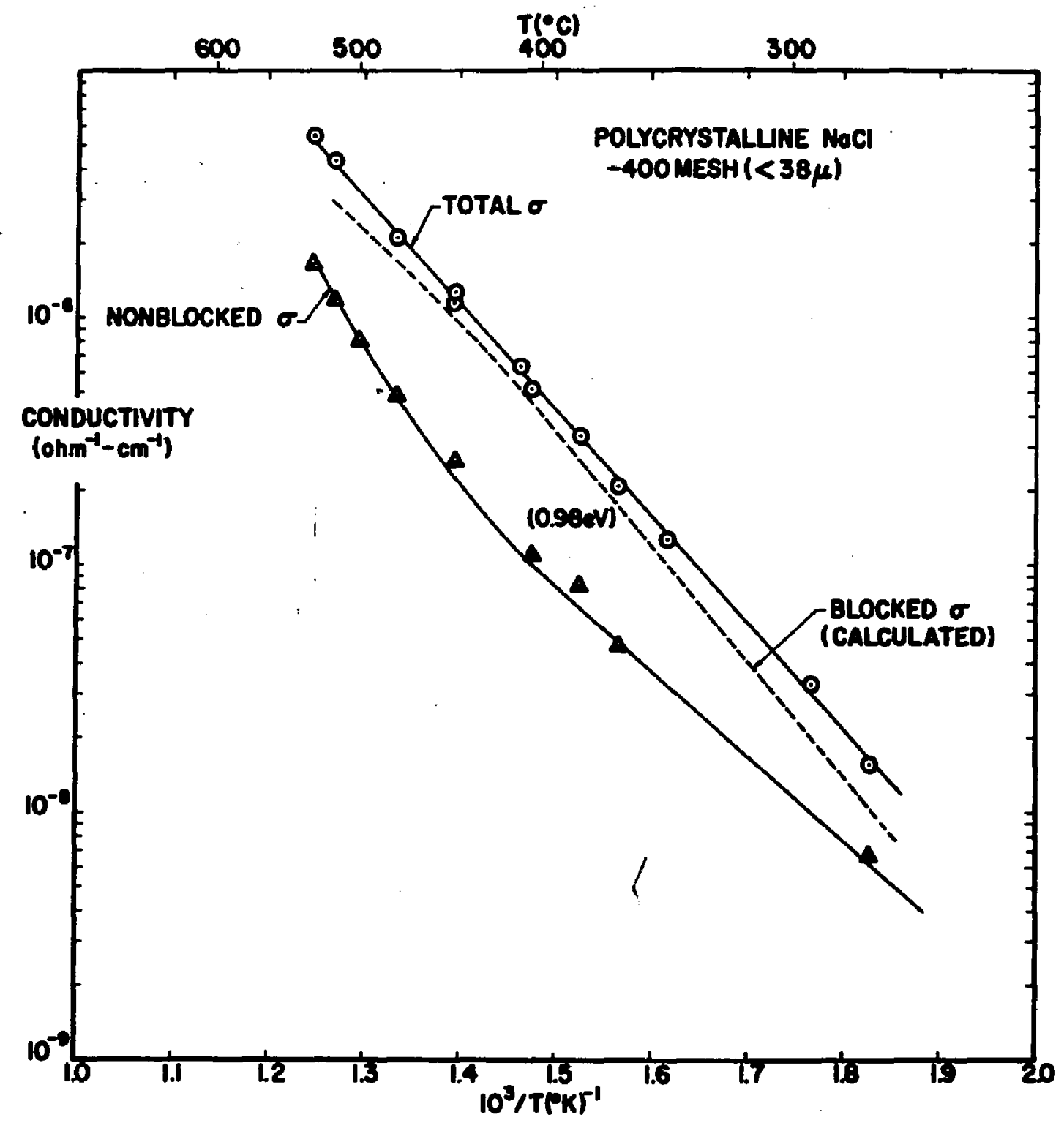

Figure 17. Conductivity versus Reciprocal Temperature for Polycrystalline Sample $-400 \mathrm{Mesh}$ 


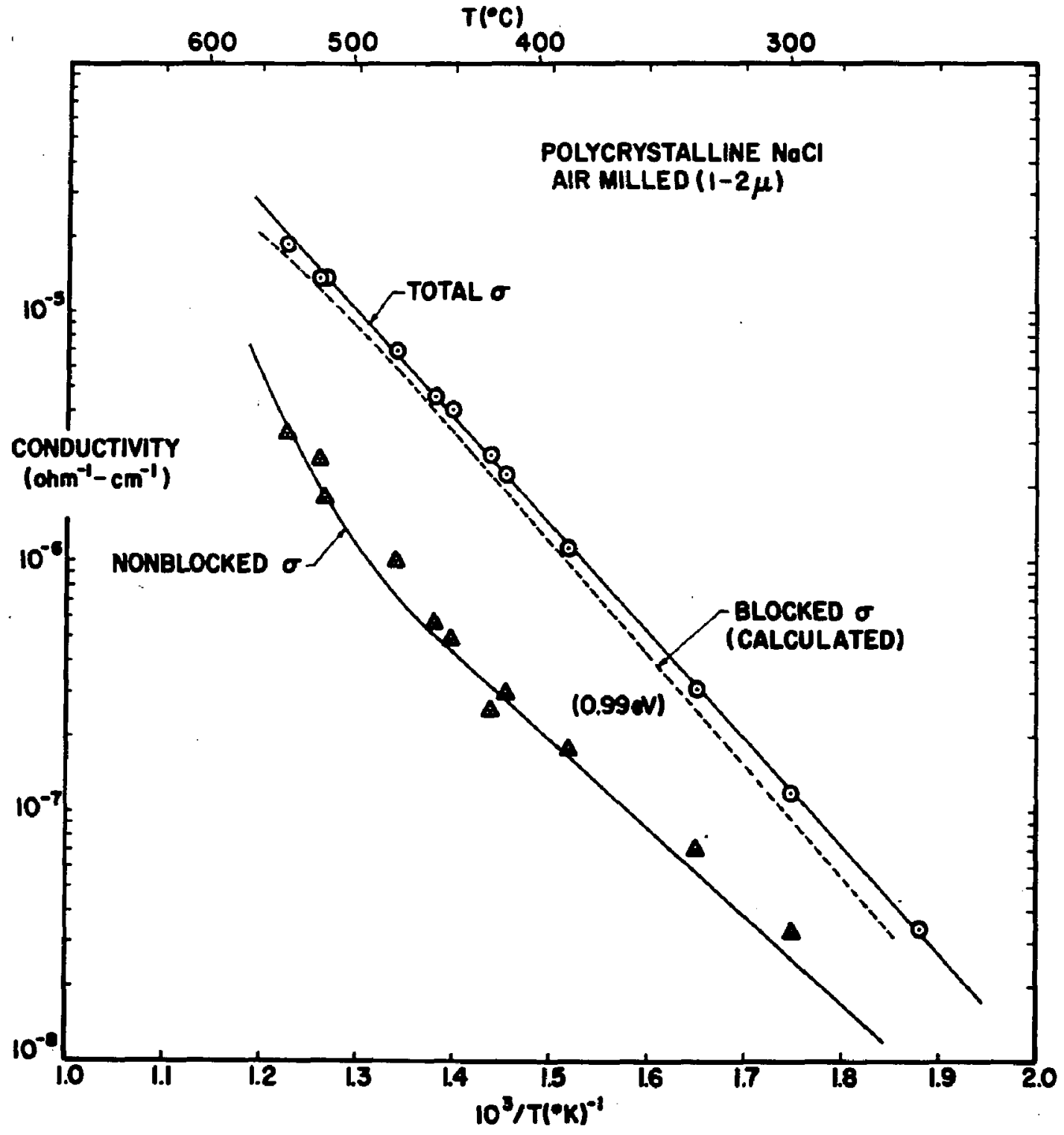

Figure 18. Conductivity versus Reciprocal Temperature for Polycrystalline Sample - Jet Milled 
steady state conductivity $\left(\sigma_{\infty}\right)$. The third line (dashed) is the calculated difference, $\sigma_{0}-\sigma_{\infty}$, i. e. the portion of the total conductivity due to the blocked carriers.

The experimental scatter in the long time points is considerably worse than for any of the other data. In most cases it was due to the drift of the zero or base line of the polarization circuity during the long times required to reach equilibrium. However, some of the scatter was undoubtably due to the samples themselves. In some cases where the experimental values were quite unstable, it was due to poor contact between the sample and the electrodes. The solid line drawn through the $\sigma_{\infty}$ points is the one ascribed to sodium vacancy conductivity in the "as-received" single crystal, i. e., referring to Figure 12, $\sigma_{0}$ in the extrinsic range and $\sigma_{\infty}$ in the intrinsic range.

Figure 19 presents the normalized curves for the data of the six fractions. Assuming that the $\sigma_{\infty}$ curve for each sample was the sodium vacancy contribution, the curves for each sample were shifted to lower conductivities until the intrinsic portion of the $\sigma_{\infty}$ curve aligned with the intrinsic curve for sodium conduction in the "as-received" crystal. No temperature shift (horizontal movement) was made since it was felt that the intrinsic conductivity at a given temperature should have been the same for all samples and that misalignment of the intrinsic curves was the result of experimental error related to the electrode-area geometry factor for the polycrystal samples. The magnitude of the shift was not systematically dependent upon particle size.

In Figure 20 the nomalized conductivities at $\sim 350^{\circ} \mathrm{C}$ are plotted as a function of particle size. While it is true that the experimental points for this diagram are taken from the conductivity plots the various samples, the curves drawn through these points do not represent the change in conductivity of the $\mathrm{NaCl}$ as a function of particle size. Rather the curves represent the change in the relative contributions of the bulk and enhanced surface layer regions to the total conductivity of the sample. 


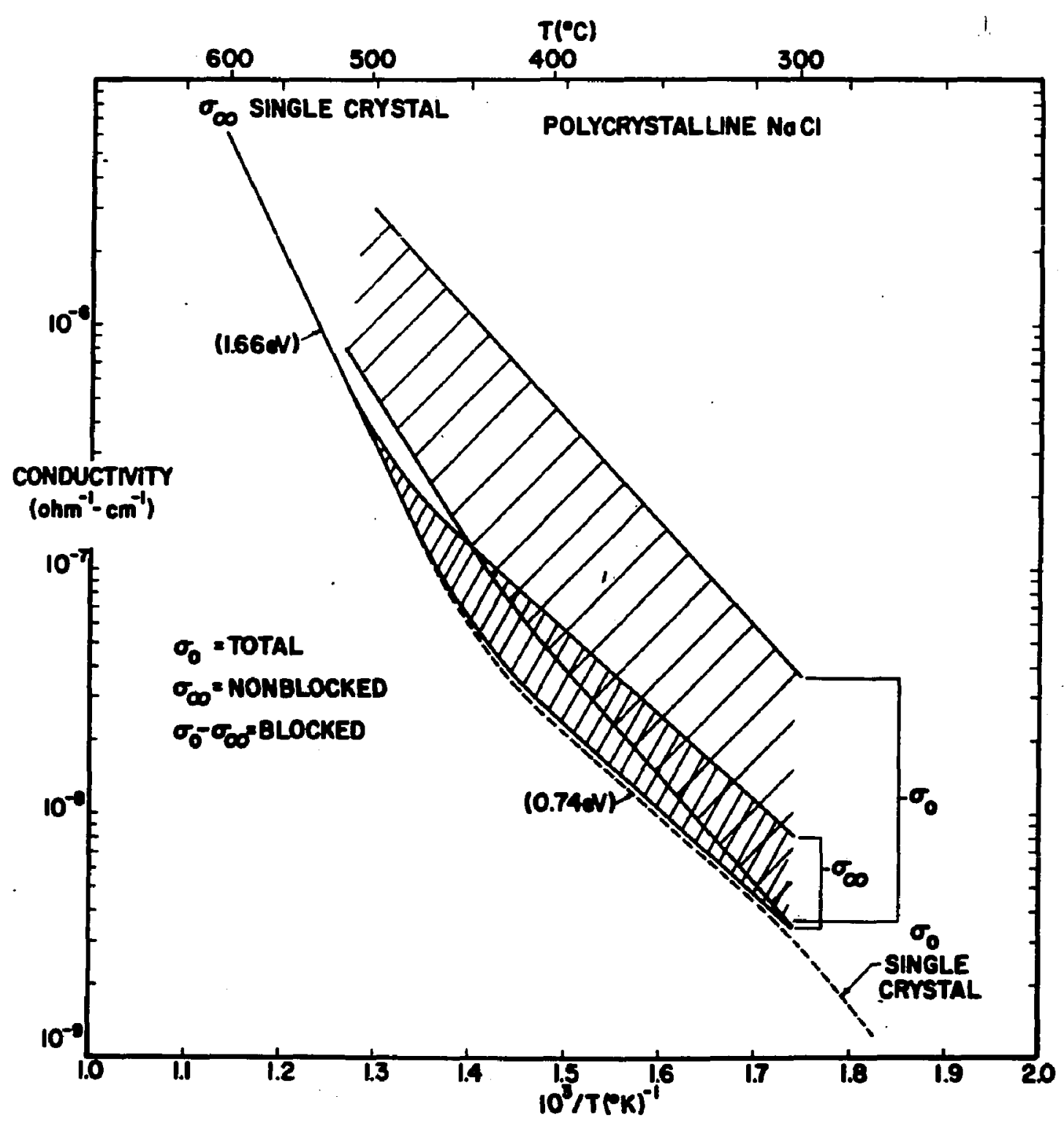

Figure 19. Summary of Polycrystalline Conductivity Results 


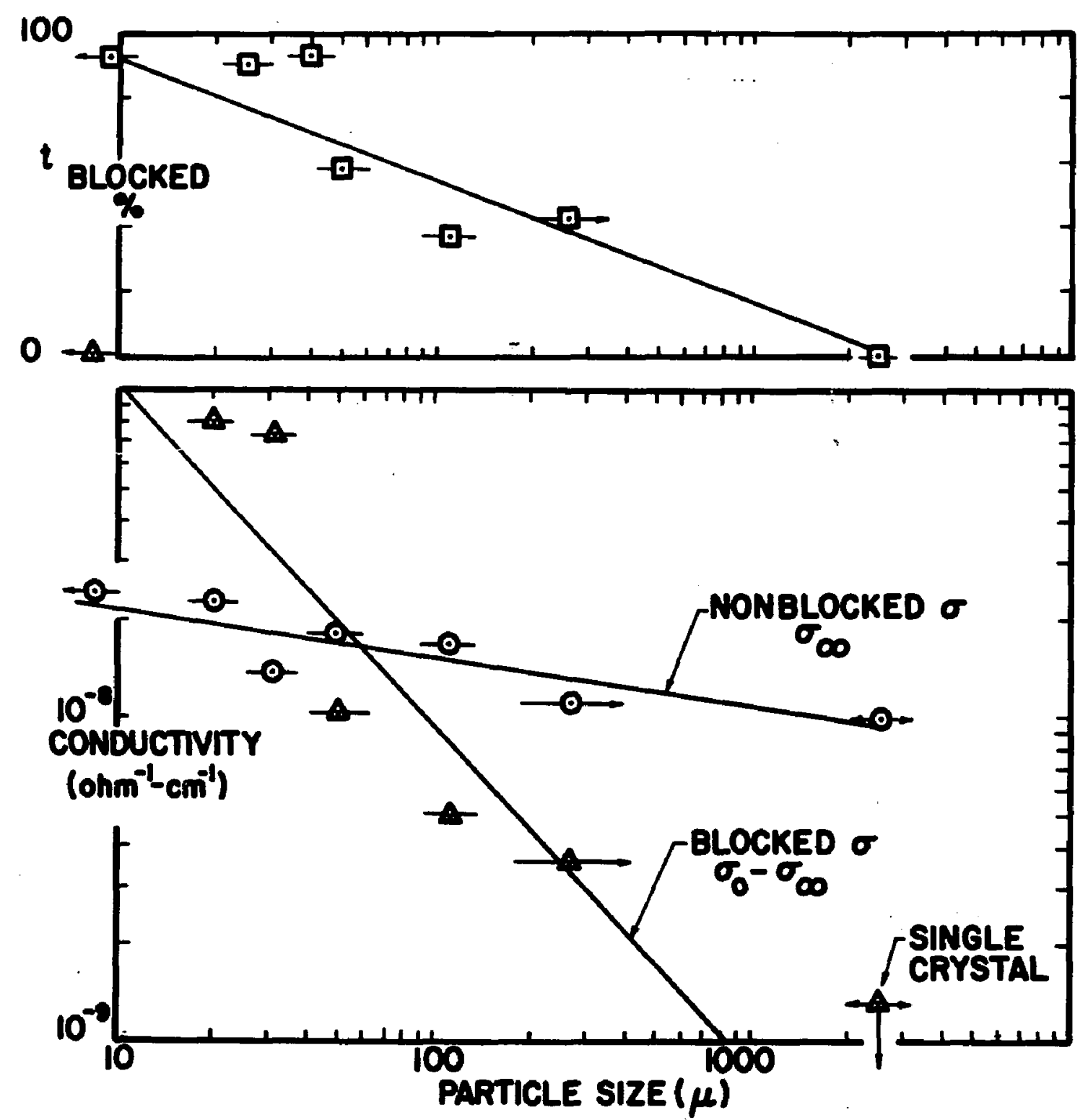

Figure 20. Conductivity and Transference Number as a Function of Particle Size 
The conductivity in the enhanced region is the same for large particles as it is for small ones, but the contribution of this region to the conductivity of the macroscopic sample is much smaller for large particles since the flux is small because of the small cross section. Therefore, the true conductivity of the enhanced region will be measured only when its conduction is more significant than the bulk conduction, i. e., when no further increase is seen in the blocked conductivity with decreasing particle size. The conductivity of the bulk region is given by the sodium vacancy conductivity for the single crystal at this temperature.

The slight particle size dependence of the sodium vacancy conduction may not be significant considering the experimental scatter and the possibility of a systematic error in the particle size study. If it were real, such a small dependence would probably not be discernible in a diffusivity study.

In contrast to this slight dependence, the dependence of the blocked carrier is quite strong. The scatter in the data points is undoubtedly partially due to the scatter in the $\sigma_{\infty}$ results. The horizontal lines or arrows with each point indicate that there is a particle size distribution within each sample and not necessarily an uncertainty in the particle size determination. If a particular particle size predominated in the fraction, the sample's conductivity would be very close to the value for that size. Therefore, some of the scatter is also the result of not knowing the effective particle size for a given sample. The blocked conductivity contribution in the single crystal is not known at this temperature but is assumed to be at least two orders of magnitude lower than the sodium vacancy contribution $\left(\sim 10^{10}\right.$ since tblocked $\sim 0$ at $\left.350^{\circ} \mathrm{C}\right)$. This would place the single crystal chlorine vacancy conductivity in reasonable agreement with the experimental values for the polycrystalline samples.

The solid line drawn through the experimental points for the blocked conductivity was calculated assuming a reciprocal particle size dependence. Since this dependence best fits the data (as opposed to $1 / \mathrm{d}^{2}$ etc.), 
the change in conductivity is proportional to the change in grain boundary area (assuming approximately equiaxed particles). If a constant boundary thickness is assumed the blocked conductivity is proportional to the grain boundary volume. This same reciprocal dependence upon particle size was found for chlorine vacancy diffusion in $\mathrm{NaCl}$ by Laurent and Bénard. ${ }^{3}$

The upper portion of Figure 20 shows the transference number for the enhanced chlorine conductivity. An evaluation of the small particle size end of this plot brings out an interesting point. Even for the smallest particle size measured $(1-2 \mu$ or less), the enhancement of the blocked conduction is still increasing. Since the contributions of the bulk and surface layer mechanisms are approximately the product of their conductivities and cross sectional areas, it is not possible to separate these two without additional information about one or the other. However, if certain assumptions are made an order of magnitude calculation can be made. Based on the transference number results, it is assumed that the contributions of the surface layer region and the bulk are about equal $\left(\sigma_{L} A_{L} / \sigma_{B} A_{B} \sim 1\right)$. If it is further assumed that the thickness of the surface layer is small with respect to the particle size, the ratio of thicknesses, $d_{L} / d_{B}$, is approximately equal to the recip rocal of conductivities for the respective regions. The conductivity of the bulk would be the single crystal sodium vacancy conductivity or about $10^{-8} \mathrm{ohm}^{-1} \mathrm{~cm}^{-1}$ at $\sim 350^{\circ} \mathrm{C}$. The conductivity of the layer region is not known and can only be estimated to be greater than $10^{-7}$ (the 1 to $2 \mu$ or less sample value) since no saturation in the.relative con ductance of the blocked carrier was seen, i.e., no indication that the particle was almost entirely made up of the layer region. Therefore, $\mathrm{d}_{\mathrm{L}} / \mathrm{d}_{\mathrm{B}} \leq \frac{10^{-8}}{10^{-7}}$ or .1 at $350^{\circ} \mathrm{C}$. An approximate maximum thickness for the layer is then set at $0.1 \mu$ at $\sim 350^{\circ} \mathrm{C}$. Lehovic ${ }^{32}$ in describing the presence of a space-charge layer due to a difference in the concentration of defects near the surface used $\mathrm{NaCl}$ as an example. The 
space charge layer thickness calculated for $\sim 300^{\circ} \mathrm{C}$ was $0.22 \mu$. Since the space charge thickness decreases with increasing temperature and the results of Figure 20 are for $\sim 350^{\circ} \mathrm{C}$, the agreement between the two layer thickness calculations is reasonable. However, it must be pointed out that Lehovic's treatment predicts on excess of cation vacancies in the layer which does not seem compatible with an enhancement of chlorine vacancy conduction. As pointed out by Lehovic, the presence of impurities may greatly alter the configuration of the space charge layer.

Since the results for the "dried" sample were quantitatively the same as the undried sample, it is felt that sufficient water was not removed to obtain the very good grain boundaries. Therefore, no deductions are made with regard to the effect of the moisture on the enhanced conductivity. However, it is noteworthy that the conductivity of sam ples pressed at 70,000 psi was the same as for samples pressed at 15, 000 psi.

The activation energies determined from the calculated curves for blocked conductivity are in reasonable agreement with proposed values for anion movement. The average value of $1.08 \mathrm{eV}$ is within the spread of experimental and theoretical values $\left(0.90^{7}\right.$ and $1.11 \mathrm{eV}^{6,33}$ respectively). Due to the larger error involved in the calculation of the blocked contribution for the larger particle sizes, it is quite probable that the average value is high and that the energy is more nearly $1.0 \mathrm{eV}$. The breakover in the calculated curves at high temperatures is thought to reflect the inability to make a meaningful subtraction of the curves rather than a true change in the slope of the anion contribution.

3. Single crystal, additively colored. Figures 21,22 and 23 pre sent the results for three single crystal samples which were additively colored so as to produce three different compositions. 


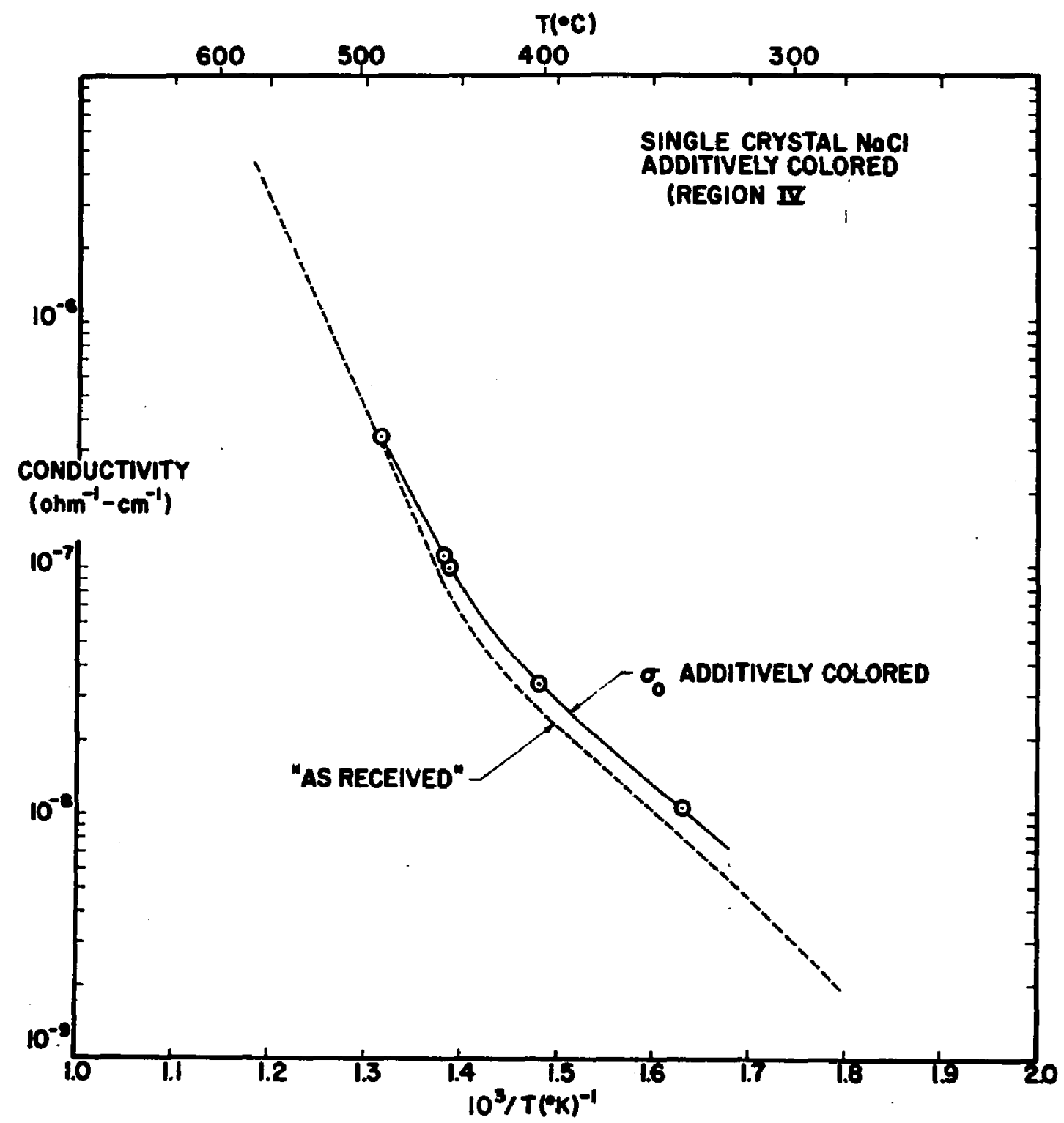

Figure 21. Conductivity versus Reciprocal Temperature for Additively Colored Single Crystal Sample - Short Treatment 


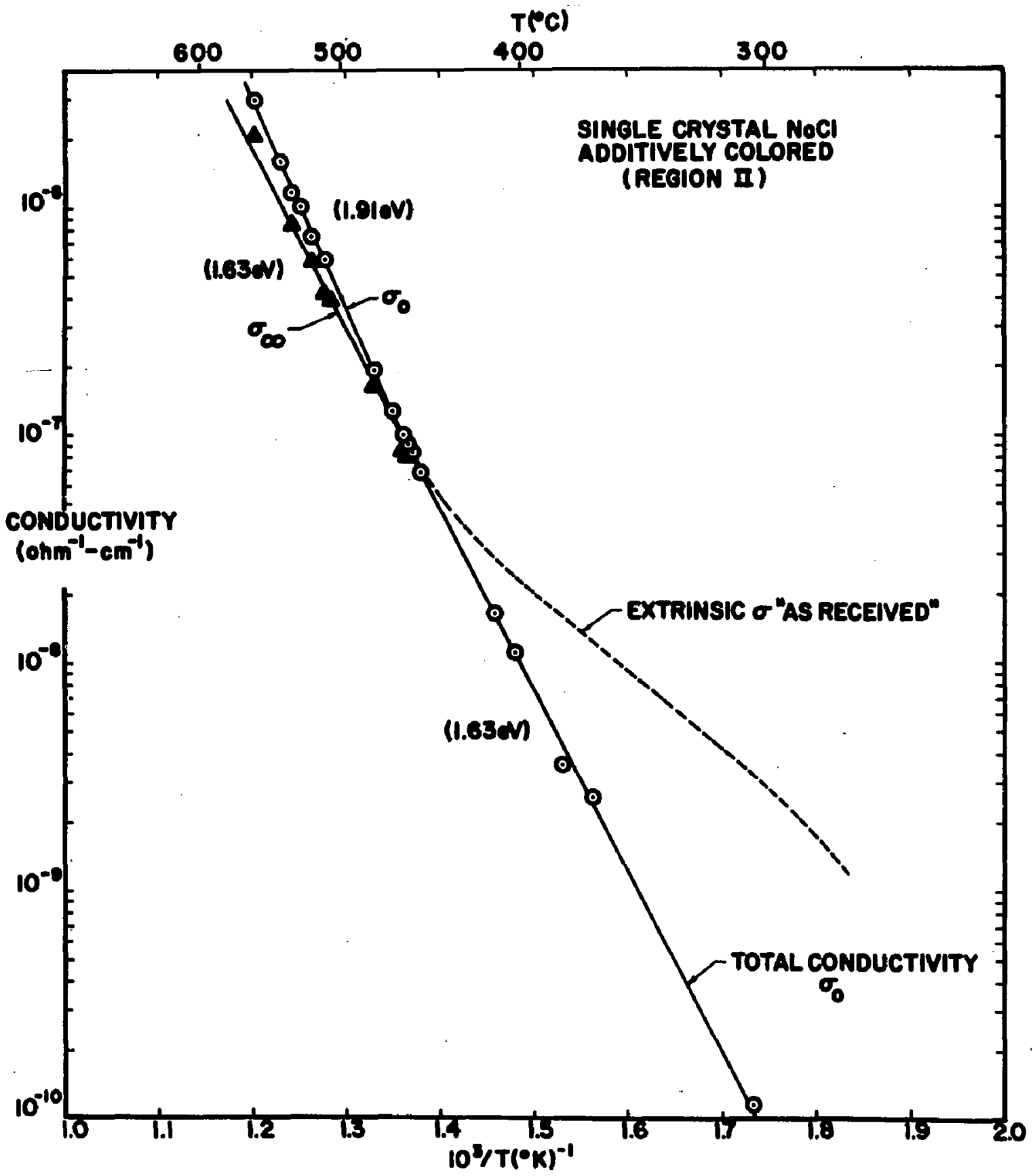

Figure 22. Conductivity versus Reciprocal Temperature for Additively Colored Single Crystal Sample - Medium Treatment 


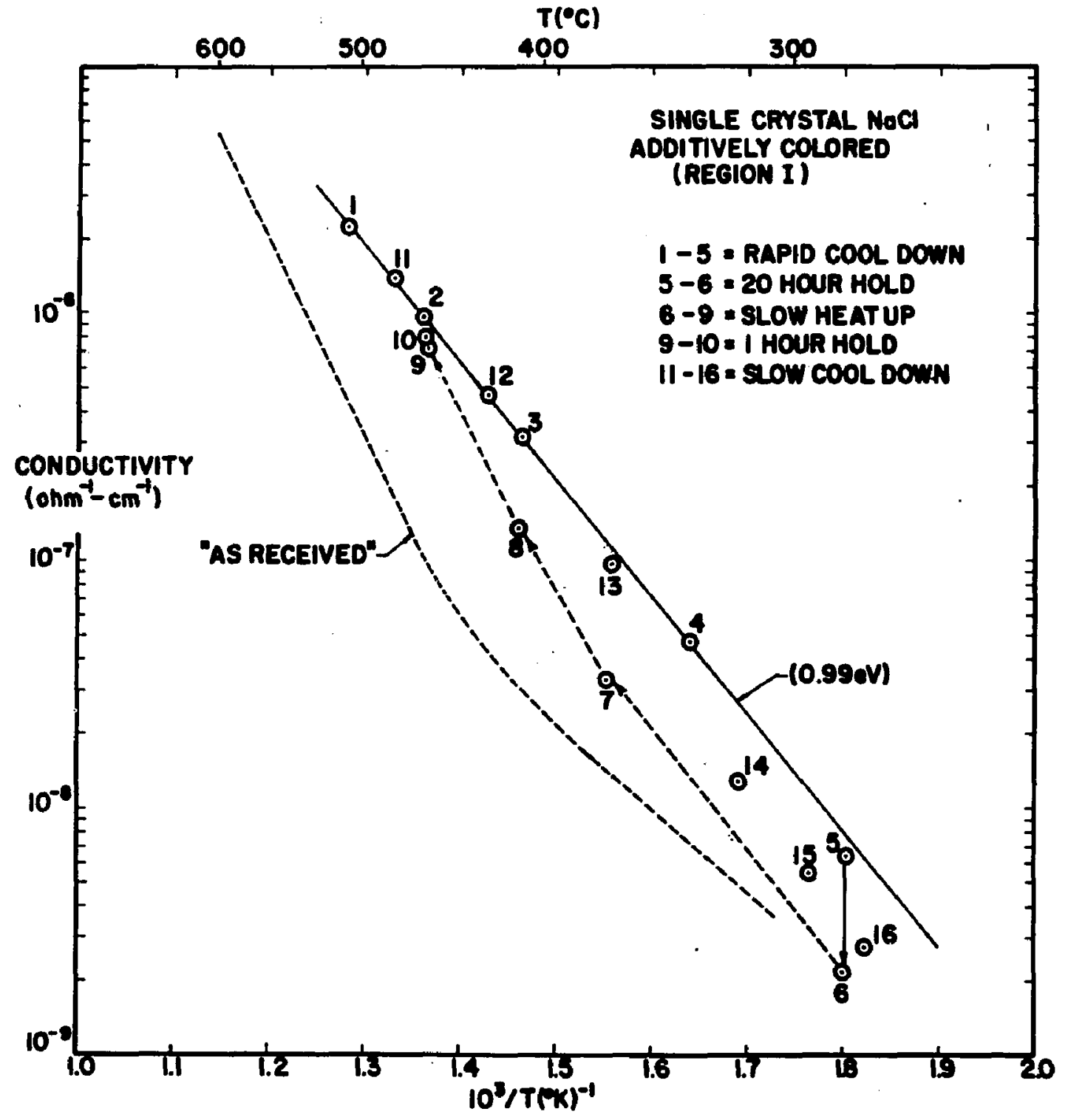

Figure 23. Conductivity versus Reciprocal Temperature for Additively 
Figure 21 presents the results of a brief study of a sample which had been held for 16 hours at $500^{\circ} \mathrm{C}$ in a sodium vapor atmosphere. Its surface appeared to have a slight yellowish tint after this reduction. That this absence of color probably indicates a lack of an appreciable color center concentration is verified by the conductivity measurements, which show no significant change in the predominent defect concentration.

Figure 22 gives the results for a crystal held at $500^{\circ} \mathrm{C}$ for 72 hours in the sodium vapor and $600^{\circ} \mathrm{C}$ for another 72 hours. Unfortunately it is impossible to say how long the sample was in a sodium vapor at $600^{\circ} \mathrm{C}$ since the top of the bomb was cracked when removed from the furnace. The sample was darkened but clear. The wire support had imbedded itself in the sample, making it necessary to grind the faces to obtain flat, parallel surfaces for electroding.

There are several important features shown by these data. The most startling is the absence of an extrinsic conductivity region as exhibited by the "as-received" crystals. Since in this region it has been well established that the sodium vacancy concentration is fixed by the con centration of divalent cation impurities, the reduction of this conduc tivity must be the result of a decrease in the concentration of the charged impurity $\left(F_{\mathrm{m}}\right)$. The absence of an extrinsic region makes it possible to follow the intrinsic curve to quite low temperature $\left(\sim 150^{\circ} \mathrm{C}\right.$ lower than normal for the "pure" single crystal). The fact that this low temperature portion of the curve is the extention of the high temperature $\sigma_{\infty}$ curve and not the $\sigma_{0}$ curve is strong evidence in favor of the high temperature steady state conductivity being $V_{\mathrm{Na}}^{\prime}$ conduction. Both the $\sigma_{o}$ and $\sigma_{\infty}$ curves are consistent with the "as-received" curves in the region where they overlap.

The results for the third sample are presented in Figure 23. This sample was held for 72 hours at $600^{\circ} \mathrm{C}$ in the sodium vapor. Upon removal from the bomb it had a clear blue surface layer (thickness about 
$1 / 8$ the cross section) and a cloudy, yellow interior. After the electrical measurements were completed, the surface layer was colorless and the interior was yellow, but clear. A subsequent treatment of 100 hours in a sulfur atmosphere at $425^{\circ} \mathrm{C}$ did not affect the $\sigma_{0}$ results but did leave the sample with only a very slightly tinted, blue interior.

It is readily seen that the results for this sample are not as well defined as were those for the other two. Even though the results are highly dependent upon the prior heating or cooling sequence, there appears to be two levels with possibly the same mechanism operating in both. Several observations may be made which add to the overall picture. First, there is the return of an extrinsic region; second, the conductivity is greater than that for the "as-received" sample; third, there appears to be a single range; and fourth, the activation energy $(0.99 \mathrm{eV})$ is comparable to that proposed for chlorine vacancy movement. ${ }^{7}, 33$

Since the results for the three samples were not readily correlated, a plot of the logarithm of the defect concentration versus the logarithm of $\mathrm{PCl}_{2}{ }^{1 / 2}$ was constructed for $\mathrm{NaCl}$ using the method outlined by $\mathrm{Kroger}$ and Vink. ${ }^{16}$ The addition of sodium ions to the crystal is the same, with respect to composition, as the loss of chlorine ions, so the normal log $\mathrm{p}_{2}{ }^{1 / 2}$ versus $\log$ concentration plot was used (Figure 24). In this method an equilibrium mass action equation is written from the formation reaction for each probable defect. For example, for vacancy pair formation in $\mathrm{NaCl}$,

$$
\mathrm{V}_{\mathrm{Na}}^{\prime}+\mathrm{V}_{\mathrm{Cl}}^{\cdot} \rightleftharpoons\left(\mathrm{V}_{\mathrm{Na}}^{\prime} \mathrm{V}_{\mathrm{C} 1}^{\cdot}\right)^{\mathbf{x}}+\mathrm{Hp}_{\mathrm{p}}
$$

and

$$
\frac{\left[\left(\mathrm{V}_{\mathrm{Na}}^{1} \mathrm{~V}_{\mathrm{Cl}}^{\cdot}\right)^{\mathrm{x}}\right]}{\left[\mathrm{V}_{\mathrm{Na}}^{1}\right]\left[\mathrm{V}_{\mathrm{Cl}}^{\cdot}\right]}=\mathrm{C} \exp \left(\mathrm{Hp}_{\mathrm{p}} / \mathrm{kT}\right)=\mathrm{Kp}
$$




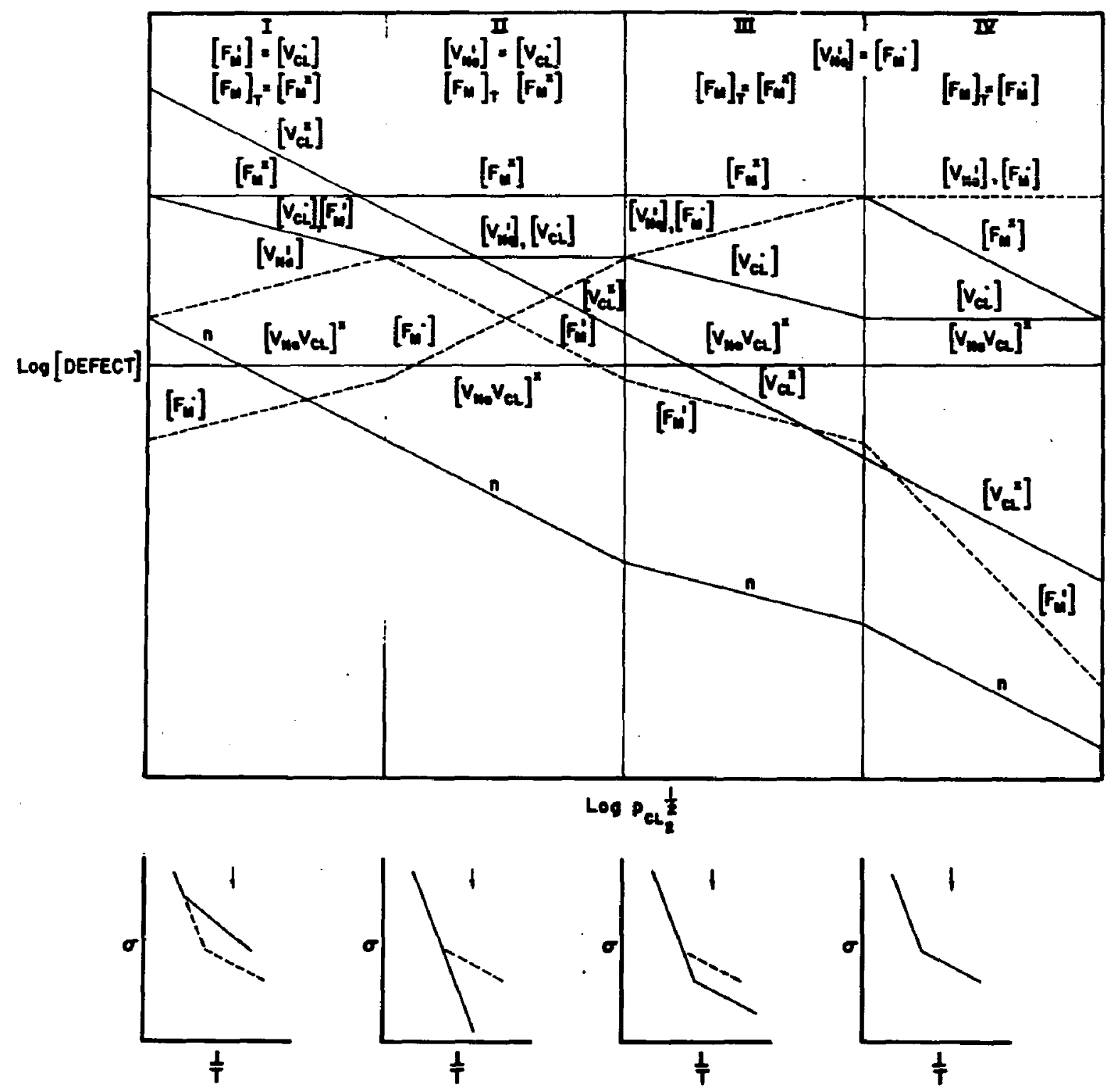

Figure 24. Logarithm of Defect Concentration versus the Logarithm of $\mathrm{PCl}_{2}^{1 / 2}$ for $\mathrm{NaCl}$ 
Since the diagram is drawn for a particular temperature, the right side of (17) is a constant, Kp. By taking the logarithm of both sides of the equation, a linear relationship is obtained:

$$
\log \left[\left(\mathrm{V}_{\mathrm{Na}}^{\prime} \mathrm{V}_{\mathrm{Cl}}^{\cdot}\right)^{\mathrm{x}}\right]-\log \left[\mathrm{V}_{\mathrm{Na}}^{\prime}\right]-\log \left[\mathrm{V}_{\mathrm{Cl}}^{\cdot}\right]=\log \mathrm{Kp}
$$

The combination of all such equations for the defects considered will result in one less equation than the number of unknowns. In order to solve for the unknowns by the method of simultaneous equations, another equation is needed which does not contain a new unknown. This equation is the equation of electrical neutrality - the sum of all of the negative charges must be equal to the sum of all the positive charges, since the overall crystal is neutral. For example, if the allowed defects are the cation vacancy, $\mathrm{V}_{\mathrm{Na}}^{\prime}$, the anion vacancy, $\mathrm{V}_{\mathrm{Cl}}$, the electron, the positive electron hole, and the vacancy pair, $\left(\mathrm{V}_{\mathrm{Na}}^{\prime} \mathrm{V}^{\cdot} \mathrm{Cl}\right)^{\mathrm{X}}$, the following equation can be written

$$
\left[\mathrm{V}_{\mathrm{Na}}^{\prime}\right]+\mathrm{n}=\left[\mathrm{V}_{\mathrm{Cl}}^{*}\right]+\mathrm{p}
$$

where $n$ and $p$ are the concentrations of the electron and hole, respectively. The vacancy pair is neutral and therefore is not included. Since the taking of the logarithm of both sides of Equation (19) will lead to a non-linear equation, the Brower approximation ${ }^{34}$ must be applied, i. e. the neutrality condition must be simplified by assuming that one negatively charged defect predominates on the left side and one positively charged defect predominates on the right. With an equal number of equations and unknowns, a simultaneous solution can be obtained. The solution must be obtained for each simplified neutrality conditions that can be written. This type of problem is easily handled by a computer and the solutions have been obtained for the compound MX. The relative positions of the defect concentrations and therefore the widths of the regions in Figure 24 will depend upon the equilibrium constants 
assumed for the mass action equations. Generally, the constants must either be determined experimentally or their relative magnitudes must be deduced by a process of trial and error in which the final selection is based upon those relative values which agree most closely with experimental results.

In constructing Figure 24 there was very little experimental information with regard to either the relative values of the constants or the widths and number of regions to be included. However, the position of each concentration is fixed in all regions once its position is arbitrarily fixed in any one region, since the pressure dependence of anydefect in any region is independent of the arbitrary selection of equilibrium constants. For convenience, the arbitrary positions of the defects were selected in such a way that the regions shown have equal widths. For example, in region II the concentrations of $\mathrm{V}_{\mathrm{Na}}^{\prime}$ and $\mathrm{V}^{*} \mathrm{Cl}$ were defined as being equal and as predominating. Therefore in regions I, III and IV the position of each is defined relative to the position in II by the pressure dependences and the fixed width of the regions.

The position of $\left[\mathrm{V}_{\mathrm{Cl}}\right]^{\mathrm{x}}$ was completely arbitrary since it does not appear in any simplified neutrality condition and there was no experimental data to establish its relative position in any region. The electron concentration was arbitrarily chosen to be low in all regions since no electronic contribution to the conductivity was seen. The concentration of vacancy pairs was placed slightly below the free chlorine vacancy concentration in region IV (the region normally encountered) in light of experimental evidence indicating this to be the case.5,6,7

As stated previously, only the predominent charged defect will make a significant contribution to the conductivity. The diagram so con structed is applicable at temperatures where the "normal" impurity controlled extrinsic conductivity is seen in "pure" $\mathrm{NaCl}$ (arrows on schematic plots of $\sigma$ versus_ $1 / T$ ). 
The following comments pertaining to each region are made to show that the experimental results can be correlated with the model represented by Figure 24 .

Region I $\left[F_{M}^{\prime}\right]=\left[\mathrm{V}_{\mathrm{Cl}}^{*}\right],\left[\mathrm{F}_{\mathrm{M}}\right]_{\mathrm{T}}=\left[\mathrm{F}_{\mathrm{M}}^{\mathrm{X}}\right]$

This region might correspond to the longest treatment and thereby to the lowest chlorine pressure. The predominent mobile defect would be the chlorine vacancy. The results presented in Figure 23 are consistent with this picture, i. $e$, the extrinsic conductivity is greater than the "as-received" sodium vacancy conductivity and the slope is within the values reported for the movement of the chlorine vacancy.

Region II $\left[\mathrm{V}_{\mathrm{Na}}^{\prime}\right]=\left[\mathrm{V}_{\mathrm{Cl}}{ }\right],\left[\mathrm{F}_{\mathrm{M}}\right]_{\mathrm{T}}=\left[\mathrm{F}_{\mathrm{M}} \mathrm{x}^{\mathrm{x}}\right]$

The neutrality condition for this region states that Schottky disorder will predominate. In this region the conductivity will be predominantly by sodium vacancy movementif $\mu_{\mathrm{V}_{\mathrm{Na}}^{1}} \gg \mu{ }^{\cdot}{ } \mathrm{Cl}$, but the magnitude will be considerably lower than for an "as-received" crystal in the extrinsic range, since the concentration of sodium vacancies is determined by the Schottky constant and not the concentration of divalent cation impurities. The experimental results for this region would be similar to Figure 22.

Region III $\left[V_{\mathrm{Na}}^{\prime}\right]=\left[F_{M}^{*}\right],\left[F_{M}\right]_{T}=\left[F_{M}^{x}\right]$

In this region the decrease of the [ $\mathrm{F}^{\circ} \mathrm{M}$ ] with $\mathrm{Na}$ addition during additive coloring brings about a corresponding decrease in [ $\left.\mathrm{V}_{\mathrm{Na}}^{\prime}\right]$. Therefore experimentally the extrinsic $V_{\mathrm{Na}}^{\prime}$ conductivity should decrease while maintaining the same activation energy. This is shown schematically in Figure 24 but was not seen experimentally. In this same region the $\left[\mathrm{V}^{\circ} \mathrm{Cl}\right]$ is seen to increase as would be expected f=om the maintainence of the Schottky product. 
$\underline{\text { Region IV }}\left[\mathrm{V}_{\mathrm{Na}}^{\prime}\right]=\left[\mathrm{F}_{\mathrm{M}}^{*}\right],\left[\mathrm{F}_{\mathrm{M}}\right]_{\mathrm{T}}=\left[\mathrm{F}_{\mathrm{M}}^{*}\right]$

This portion of the diagram shows the defect concentrations as they are normally encountered in $\mathrm{NaCl}$. The extrinsic sodium vacancy conductivity is controlled by a constant divalent cation impurity concentration, and the chlorine vacancy concentration is inversely proportional to the impurity content. The concentration of vacancy pairs, in the position shown, would be expected to contribute to chlorine diffusivity since the mobility of a pair is generally greater than that of the free vacancy. The concentration of pairs will be pressure insensitive since its mass action equation (Equation 17) is

$$
\left[\begin{array}{ll}
\mathrm{V}_{\mathrm{Na}}^{\prime} & \mathrm{V}_{\mathrm{Cl}}^{\cdot}
\end{array}\right]^{\mathrm{x}}=\mathrm{K}_{\mathrm{p}}^{\prime}
$$

since

$$
\left[\mathrm{V}_{\mathrm{Na}}^{\prime}\right]\left[\mathrm{V}_{\mathrm{Cl}}^{\cdot}\right]=\mathrm{K}_{\mathrm{s}} \text { and } \mathrm{K}_{\mathrm{s}} \mathrm{K}_{\mathrm{p}}=\mathrm{K}_{\mathrm{P}}^{\prime}
$$

where $\mathrm{K}_{\mathrm{s}}$ = the Schottky constant.

The fact that there is a finite width to this particular nonstoichiometric region, with respect to the non-pressure dependence of the $\left[\mathrm{F}^{\cdot} \mathrm{M}\right]$, explains the results of Figure 21 where the crystal was affected very little by a small amount of reduction. A finite width to region II would also explain how one might expect to stop the reduction at a condition where the concentrations of cation and anion vacancies are equal. A very interesting possible experimental technique is suggested by the results for the additively colored crystals. Since many systems have regions of intrinsic conductivity which are very limited with respect to their temperature range, the ability to expand such a region by reducing the concentration of charged impurities through adjustment of the composition, would be very helpful in determining a precise value of the activation energy. 


\section{SUMMARY}

In "pure" single crystal $\mathrm{NaCl}$, at high temperature, the total conductivity was found to be the sum of two contributions - a steady state conductivity $\sigma_{\infty}$, and a blocked conductivity $\sigma_{0}-\sigma_{\infty}$. At $600^{\circ} \mathrm{C}$ the blockdefect current contributed as much as $35 \%$ of the total conductivity.

The results for a sample in which the composition had been altered by adding an excess of sodium ions to the lattice, showed the same polarization with the added feature that the extrinsic, impurity controlled conductivity was not present. The low temperature portion of the intrinsic curve, as revealed by the absence of the extrinsic curve, was the extension of the high temperature steady state curve $\left(\sigma_{\infty}\right)$ and not the total conductivity curve. The increased length of the intrinsic curve made it possible to accurately determine the intrinsic activation energy. The energy determined was $1.63 \mathrm{eV}$, not the accepted $1.86 \mathrm{eV}$ (which was the $\sigma_{O}$ value determined for the "as-received" single crystal by the author). Assuming the $1.63 \mathrm{eV}$ to be the sum of the energy for movement plus one half the energy of formation, the Schottky energy determined will be lower by $\sim 0.4 \mathrm{eV}$ than one determined using $1.86 \mathrm{eV}$. If it is true that the Schottky energy is indeed lower. by this much, the energies calculated for the movement of the chlorine vacancy using the higher value will be $\sim 0.2 \mathrm{eV}$ too low.

The measurement of the conductivities of polycrystalline samples as a function of both temperature and particle size presented some additional insight into the polarization mechanism. The contribution of the 
blocked carrier to the total conductivity of a sample was found to increase inversely with the particle size. The steady state contribution remained nearly constant. In the light of the results of Laurent and. Bénard, who found a reciprocal dependence on particle size for chlorine diffusion and no dependence for sodium diffusion, it is concluded that the blocked carrier is the chlorine defect and the non-blocked is the sodium defect. The identity of the chlorine defect is not certain but it would appear to be the free chlorine vacancy, as opposed to the neutral vacancy pair, since in most cases only charged defects are expected to contribute to the electrical conductivity.

While the contribution of the chlorine conduction to the total was about $90 \%$ for a particle size of approximately 1 micron, an order of magnitude calculation placed the enhanced layer thickness at $0.1 \mathrm{mi-}$ cron or less. While this is in agreement with Lehovic's estimate of the thickness of the space charge layer in $\mathrm{NaCl}$ at this temperature, the excess of cation vacancy required in this layer does not seem compatible with the enhancement of chlorine vacancy movement.

In conclusion, for $\mathrm{NaCl}$ it may be said that -

1. The single crystal a. c. conductivity at high temperatures is the sum of the contributions of two ionic carriers;

2. The blocked carrier is the chlorine vacancy and its conduction is reciprocally dependent upon particle size;

3. The non-blocked carrier is the sodium vacancy and its conduction is nearly independent of particle size;

4. The contribution of the chlorine vacancy to the total conductivity is significant in the intrinsic range, even for single crystals;

5. The enhanced layer thickness is less than $0.1 \mu$ at $350^{\circ} \mathrm{C}$;

6. Based on the proposed model, the accepted energy for intrinsic sodium vacancy conduction is too high, thereby making the calculated Schottky energy too high by as much as $0.4 \mathrm{eV}$. 


\section{APPENDIX}

\section{TABLE 3}

Data for "as-received" single crystal sample presented in Figure 12 $t / A=0.585 \mathrm{~cm}^{-1}$

\begin{tabular}{lll}
$\begin{array}{c}\text { Temperature } \\
\left({ }^{\circ} \mathrm{C}\right)\end{array}$ & $\begin{array}{c}\text { Conductivity } \\
\left(\mathrm{ohm}^{-1} \mathrm{~cm}^{-1}\right)\end{array}$ & Remarks \\
\hline 472 & $1.58 \times 10^{-7}$ & Heating \\
484 & 2.26 & \\
494 & 3.25 & \\
504 & 4.45 & \\
470 & 1.57 & \\
460 & 1.15 & \\
451 & $8.78 \times 10^{-8}$ & Held overnight \\
443 & 6.96 & Cooling \\
421 & 3.86 & \\
414 & 3.35 & \\
392 & 2.21 & \\
383 & 1.83 & \\
374 & 1.57 & \\
365 & 1.32 & \\
352 & 1.04 & \\
338 & $7.78 \times 10^{-9}$ & \\
329 & 6.38 & \\
& &
\end{tabular}


Table 3 (Contd.)

\begin{tabular}{|c|c|c|}
\hline $\begin{array}{l}\text { Temperature } \\
\left({ }^{\circ} \mathrm{C}\right)\end{array}$ & $\begin{array}{l}\text { Conductivity } \\
\left(\mathrm{ohm}^{-1} \mathrm{~cm}^{-1}\right)\end{array}$ & Remarks \\
\hline 316 & 4.77 & \multirow{14}{*}{ Heating } \\
\hline 298 & 3.01 & \\
\hline 287 & 2.18 & \\
\hline 278 & 1.64 & \\
\hline 269 & 1.18 & \\
\hline 226 & $1.77 \times 10^{-10}$ & \\
\hline 193 & $2.72 \times 10^{-11}$ & \\
\hline 226 & $1.73 \times 10^{-10}$ & \\
\hline 233 & 2.43 & \\
\hline 238 & 3.25 & \\
\hline 248 & 4.91 & \\
\hline 255 & 6.84 & \\
\hline 217 & 1.06 & \\
\hline 209 & $6.44 \times 10^{-11}$ & \\
\hline \multicolumn{3}{|c|}{$t / A=0.902 \mathrm{~cm}^{-1}$} \\
\hline $\begin{array}{c}\text { Temperature } \\
\left({ }^{\circ} \mathrm{C}\right)\end{array}$ & $\begin{array}{l}\text { Conductivity } \\
\left(\mathrm{ohm} \mathrm{m}^{-1} \mathrm{~cm}^{-1}\right)\end{array}$ & Remarks \\
\hline 411 & $3.76 \times 10^{-8}$ & \multirow{3}{*}{$\begin{array}{l}\text { Same sample, new electrodes } \\
\text { Heating. }\end{array}$} \\
\hline 458 & $1.20 \times 10^{-7}$ & \\
\hline 479 & $2.38 \times 10^{-7}$ & \\
\hline 525 & $1.05 \times 10^{-6}$ & \multirow[t]{3}{*}{ Polarization present } \\
\hline 549 & 2.26 & \\
\hline 570 & 4.24 & \\
\hline
\end{tabular}


Table 3 (Contd.)

\begin{tabular}{cll}
\hline $\begin{array}{c}\text { Temperature } \\
\left({ }^{\circ} \mathrm{C}\right)\end{array}$ & $\begin{array}{c}\text { Conductivity } \\
\left(0 \mathrm{oh}^{-1} \mathrm{~cm}^{-1}\right)\end{array}$ & \multicolumn{1}{c}{ Remarks -} \\
460 & $1.22 \times 10^{-7}$ & $\sigma_{\mathrm{O}}$, overnight \\
& $1.05 \times 10^{-7}$ & $\sigma_{\infty}, \mathrm{t}_{\mathrm{B}}=14 \%(11 / 2 \mathrm{Hrs})$. \\
500 & $4.64 \times 10^{-7}$ & $\sigma_{\mathrm{O}}$ \\
& $3.77 \times 10^{-7}$ & $\sigma_{\infty}, \mathrm{t}_{\mathrm{B}}=19 \%(3 \mathrm{Hrs})$. \\
\hline
\end{tabular}

\section{TABLE 4}

Data for +100 mesh polycrystalline sample presented in Figure 13

$$
t / A=0.436 \mathrm{~cm}^{-1}
$$

\begin{tabular}{|c|c|c|}
\hline $\begin{array}{c}\text { Temperature } \\
\left({ }^{\circ} \mathrm{C}\right)\end{array}$ & $\begin{array}{l}\text { Conductivity } \\
\left(\mathrm{ohm}^{-1} \mathrm{~cm}^{-1}\right)\end{array}$ & Remarks \\
\hline 275 & $7.06 \times 10^{-9}$ & Overnight \\
\hline 292 & $1.89 \times 10^{-8}$ & Heating \\
\hline 330 & 4.93 & \\
\hline 352 & 8.02 & \\
\hline 373 & $1.21 \times 10^{-7}$ & \\
\hline 414 & 2.54 & \\
\hline 472 & 7.28 & \\
\hline 517 & $1.73 \times 10^{-6}$ & \\
\hline 537 & 2.69 & \\
\hline \multirow[t]{2}{*}{551} & $3.61 \times 10^{-6}$ & $\sigma_{\circ}$ \\
\hline & $2.64 \times 10^{-6}$ & $\sigma_{\infty}, t_{B}=27 \%(1 / 2 \mathrm{Hr})$. \\
\hline \multirow[t]{2}{*}{536} & $2.44 \times 10^{-6}$ & $\sigma_{\mathrm{o}}$ \\
\hline & $1.73 \times 10^{-6}$ & $\sigma_{\infty}, \mathrm{t}_{\mathrm{B}}=29 \%(1 / 2 \mathrm{Hr})$. \\
\hline \multirow[t]{2}{*}{507} & $1.18 \times 10^{-6}$ & $\sigma_{\mathrm{o}}$ \\
\hline & $8.33 \times 10^{-7}$ & $\sigma_{\infty}, t_{B}=29 \%(3 / 4 \mathrm{Hr})$. \\
\hline
\end{tabular}


- Table 4 (Contd.)

\begin{tabular}{|c|c|c|}
\hline $\begin{array}{l}\text { Temperature } \\
\left({ }^{\circ} \mathrm{C}\right)\end{array}$ & $\begin{array}{l}\text { Conductivity } \\
\left(\text { ohm } \mathrm{m}^{-1} \mathrm{~cm}^{-1}\right)\end{array}$ & Remarks \\
\hline \multirow[t]{2}{*}{462} & $3.90 \times 10^{-7}$ & $\sigma_{\mathrm{o}}$ \\
\hline & $2.64 \times 10^{-7}$ & $\sigma_{\infty}, t_{B}=32 \%(1 \mathrm{Hr})$. \\
\hline \multirow[t]{2}{*}{437} & $2.10 \times 10^{-7}$ & $\sigma_{0}$ \\
\hline & $1.51 \times 10^{-7}$ & $\sigma_{\infty}, t_{B}=28 \%(1 \mathrm{Hr})$. \\
\hline \multirow[t]{2}{*}{389} & $6.54 \times 10^{-8}$ & $\sigma_{O}$, overnight \\
\hline & $4.22 \times 10^{-8}$ & $\sigma_{\infty}, t_{B}=35 \%(21 / 4 \mathrm{Hrs})$. \\
\hline \multirow[t]{2}{*}{330} & $1.38 \times 10^{-8}$ & $\sigma_{\mathrm{o}}$ \\
\hline & $1.18 \times 10^{-8}$ & $\sigma_{\infty}, t_{B}=14 \%(21 / 2 \mathrm{Hrs})$. \\
\hline 292 & $4.80 \times 10^{-9}$ & Cooling \\
\hline
\end{tabular}

TABLE 5

Data for $-100+200$ mesh polycrystalline sample presented in Figure 14

$$
t / A=0.523 \mathrm{~cm}^{-1}
$$

$\begin{array}{cll}\begin{array}{c}\text { Temperature } \\ \left({ }^{\circ} \mathrm{C}\right)\end{array} & \begin{array}{l}\text { Conductivity } \\ \left(\text { ohm } \mathrm{cm}^{-1}\right)\end{array} & \text { Remarks } \\ 294 & 1.90 \times 10^{-8} & \begin{array}{c}\text { Overnight } \\ \text { Heating }\end{array} \\ 312 & 2.98 & \text { Held } \\ 351 & 4.73 & \text { Heating } \\ 397 & 8.47 & \\ 447 & 1.86 \times 10^{-7} & \\ 467 & 4.81 & \\ 511 & 7.01 & \\ 545 & 1.70 \times 10^{-6} & \\ 555 & 3.71 & \\ 560 & 4.76 & \end{array}$


Table 5 (Contd.)

\begin{tabular}{|c|c|c|}
\hline $\begin{array}{c}\text { Temperature } \\
\left({ }^{\circ} \mathrm{C}\right)\end{array}$ & $\begin{array}{l}\text { Conductivity } \\
\left(0 h \mathrm{~m}^{-1} \mathrm{~cm}^{-1}\right)\end{array}$ & Remarks \\
\hline 541 & 3.45 & Cooling \\
\hline 522 & 2.12 & \\
\hline 507 & 1.53 & \\
\hline 494 & 1.14 & \\
\hline \multirow[t]{2}{*}{432} & $2.68 \times 10^{-7}$ & $\sigma_{\mathrm{o}}$ \\
\hline & $1.53 \times 10^{-7}$ & $\sigma_{\infty}, t_{B}=43 \%(1 / 2 \mathrm{Hr})$. \\
\hline 450 & 3.82 & Heating \\
\hline 497 & $1.14 \times 10^{-6}$ & \\
\hline 475 & $7.17 \times 10^{-7}$ & Cooling \\
\hline 453 & 4.31 & \\
\hline 425 & 2.35 & \\
\hline 397 & 1.27 & \\
\hline 353 & 4.45 & \\
\hline 258 & $2.48 \times 10^{-9}$ & Overnight, no polarization \\
\hline 230 & $8.37 \times 10^{-10}$ & \\
\hline 193 & 1.67 & A fast time polarization \\
\hline 233 & 9.00 & Heating \\
\hline 379 & $7.64 \times 10^{-8}$ & Some long time polarization \\
\hline 392 & $1.01 \times 10^{-7}$ & \\
\hline 426 & 2.19 & \\
\hline 469 & 5.75 & \\
\hline 483 & 8.16 & \\
\hline \multirow[t]{2}{*}{490} & 9.52 & $\sigma_{\mathrm{o}}$ \\
\hline & 5.49 & $\sigma_{\infty}, t_{B}=43 \%(1 / 4 \mathrm{Hr})$. \\
\hline \multirow[t]{2}{*}{422} & $1.80 \times 10^{-7}$ & $\sigma_{\mathrm{o}}$ \\
\hline & $1.04 \times 10^{-7}$ & $\sigma_{\infty}, t_{B}=42 \%(1 \mathrm{Hr})$. \\
\hline
\end{tabular}


Table 5 (Contd.)

\begin{tabular}{cll}
$\begin{array}{c}\text { Temperature } \\
\left({ }^{\circ} \mathrm{C}\right)\end{array}$ & $\begin{array}{c}\text { Conductivity } \\
\left(\mathrm{ohm} \mathrm{m}^{-1} \mathrm{~cm}^{-1}\right)\end{array}$ & \multicolumn{1}{c}{ Remarks } \\
\hline 474 & $6.28 \times 10^{-7}$ & $\sigma_{\mathrm{O}}$ \\
& $3.66 \times 10^{-7}$ & $\sigma_{\infty}, \mathrm{t}_{\mathrm{B}}=42 \%(1 / 2 \mathrm{Hr})$. \\
547 & $3.51 \times 10^{-6}$ & $\sigma_{\mathrm{O}}$ \\
& $2.34 \times 10^{-6}$ & $\sigma_{\mathrm{OO}}, \mathrm{t}_{\mathrm{B}}=33 \%(1 / 3 \mathrm{Hr})$. \\
\hline
\end{tabular}

\section{TABLE 6}

Data for $-200+325$ mesh polycrystalline sample presented in Figure 15

$$
t / A=0.319 \mathrm{~cm}^{-1}
$$

\begin{tabular}{|c|c|c|}
\hline $\begin{array}{c}\text { Temperature } \\
\left({ }^{\circ} \mathrm{C}\right)\end{array}$ & $\begin{array}{l}\text { Conductivity } \\
\left(\text { ohm }^{-1} \mathrm{~cm}^{-1}\right)\end{array}$ & Remarks \\
\hline 251 & $8.55 \times 10^{-9}$ & Heating \\
\hline 311 & $5.17 \times 10^{-8}$ & \\
\hline 334 & 9.25 & \\
\hline 371 & $2.14 \times 10^{-7}$ & Some polarization \\
\hline 408 & 4.56 & \\
\hline 473 & $1.56 \times 10^{-6}$ & \\
\hline 526 & 3.83 & 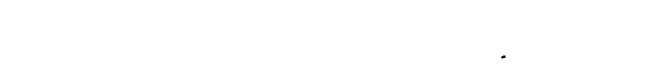 \\
\hline \multirow[t]{2}{*}{423} & $3.89 \times 10^{-7}$ & $\sigma_{\mathrm{O}}$, overnight \\
\hline & $1.57 \times 10^{-7}$ & $\sigma_{\infty}, t_{B}=60 \%(11 / 4$ Hrs. $)$ \\
\hline \multirow[t]{2}{*}{449} & $7.02 \times 10^{-7}$ & $\sigma_{\mathrm{O}}$ \\
\hline & $3.06 \times 10^{-7}$ & $\sigma_{\infty}, t_{B}=56 \%(3 / 4 \mathrm{Hr})$. \\
\hline \multirow[t]{2}{*}{485} & $1.48 \times 10^{-6}$ & $\sigma_{\mathrm{o}}$ \\
\hline & $7.02 \times 10^{-7}$ & $\sigma_{\infty}, t_{B}=53 \%(1 \mathrm{Hr})$. \\
\hline \multirow[t]{2}{*}{513} & $2.58 \times 10^{-6}$ & $\sigma_{0}$ \\
\hline & $1.39 \times 10^{-6}$ & $\sigma_{\infty}, t_{B}=46 \%(1 / 2 \mathrm{Hr})$. \\
\hline
\end{tabular}


Table 6 (Contd.)

\begin{tabular}{|c|c|c|}
\hline $\begin{array}{l}\text { Temperature } \\
\left({ }^{\circ} \mathrm{C}\right)\end{array}$ & $\begin{array}{l}\text { Conductivity } \\
\left(\mathrm{ohm}^{-1} \mathrm{~cm}^{-1}\right)\end{array}$ & Remarks \\
\hline 422 & $3.48 \times 10^{-7}$ & Overnight \\
\hline 450 & $6.32 \times 10^{-7}$ & Heating \\
\hline 488 & $1.46 \times 10^{-6}$ & \\
\hline 501 & 1.94 & \\
\hline 519 & 2.78 & \\
\hline 541 & 4.34 & \\
\hline \multirow[t]{2}{*}{551} & $5.26 \times 10^{-6}$ & $\sigma_{\mathrm{o}}$ \\
\hline & $3.41 \times 10^{-6}$ & $\sigma_{\infty}, t_{B}=35 \%(1 / 2 \mathrm{Hr})$. \\
\hline 536 & $3.83 \times 10^{-6}$ & Cooling \\
\hline 507 & 2.07 & \\
\hline 490 & 1.44 & \\
\hline 478 & 1.10 & \\
\hline \multirow[t]{2}{*}{412} & $2.61 \times 10^{-7}$ & $\sigma_{0}$ \\
\hline & $1.38 \times 10^{-7}$ & $\sigma_{\infty}, t_{B}=53 \%(3 / 4 \mathrm{Hr})$. \\
\hline 396 & $1.82 \times 10^{-7}$ & Cooling \\
\hline 373 & $9.83 \times 10^{-8}$ & \\
\hline 341 & -4.79 & \\
\hline 326 & 3.14 & \\
\hline
\end{tabular}




\section{TABLE 7}

Data for $-325+400$ mesh polycrystalline sample presented in Figure 16 $t / A=0.246 \mathrm{~cm}^{-1}$

\begin{tabular}{|c|c|c|}
\hline $\begin{array}{c}\text { Temperature } \\
\left.\qquad{ }^{\circ} \mathrm{C}\right)\end{array}$ & $\begin{array}{l}\text { Conductivity } \\
\left(\mathrm{ohm}^{-1} \mathrm{~cm}^{-1}\right)\end{array}$ & Remarks \\
\hline 348 & $2.61 \times 10^{-7}$ & Overnight \\
\hline 358 & 3.27 & \\
\hline 396 & 7.70 & \\
\hline 438 & $1.87 \times 10^{-6}$ & \\
\hline 504 & 5.76 & \\
\hline \multirow[t]{2}{*}{531} & $8.59 \times 10^{-6}$ & $\sigma_{0}$ \\
\hline & $3.47 \times 10^{-6}$ & $\sigma_{\infty}, t_{B}=60 \%(1 / 3 \mathrm{Hr})$. \\
\hline 423 & $\begin{array}{l}1.13 \times 10^{-6} \\
2.41 \times 10^{-7}\end{array}$ & $\begin{array}{l}\sigma_{0} \\
\sigma_{\infty}, t_{B}=79 \%(1 \mathrm{Hr} .)\end{array}$ \\
\hline 466 & $2.51 \times 10^{-6}$ & Heating \\
\hline 501 & 4.70 & \\
\hline 523 & 7.13 & \\
\hline 541 & 9.94 & \\
\hline 561 & $1.16 \times 10^{-5}$ & \\
\hline \multirow[t]{2}{*}{563} & $1.18 \times 10^{-5}$ & $\sigma_{0}$ \\
\hline & $3.94 \times 10^{-6}$ & $\sigma_{\infty}, t_{B}=67 \%(1 / 3 \mathrm{Hr})$. \\
\hline 437 & $1.29 \times 10^{-6}$ & Overnight \\
\hline \multirow[t]{2}{*}{473} & $2.56 \times 10^{-6}$ & $\sigma_{\mathrm{o}}$ \\
\hline & $7.04 \times 10^{-7}$ & $\sigma_{\infty}, t_{B}=72 \%(3 / 4 \mathrm{Hr})$. \\
\hline 511 & $5.54 \times 10^{-6}$ & \\
\hline
\end{tabular}


TABLE 8

Data for -400 mesh polycrystalline sample presented in Figure 17

$$
t / A=0.266 \mathrm{~cm}^{-1}
$$

\begin{tabular}{|c|c|c|}
\hline $\begin{array}{c}\text { Temperature } \\
\left({ }^{\circ} \mathrm{C}\right)\end{array}$ & $\begin{array}{l}\text { Conductivity } \\
\left(\mathrm{ohm}^{-1} \mathrm{~cm}^{-1}\right)\end{array}$ & Remarks \\
\hline 341 & $1.86 \times 10^{-7}$ & Heating \\
\hline 362 & 3.00 & Heating \\
\hline 387 & 5.40 & Heating \\
\hline 416 & 9.71 & Heating \\
\hline 432 & $1.27 \times 10^{-6}$ & Heating \\
\hline 455 & 1.95 & Heating \\
\hline 490 & 3.46 & Heating \\
\hline \multirow[t]{2}{*}{499} & $3.64 \times 10^{-6}$ & $\sigma_{\mathrm{o}}$ \\
\hline & $7.87 \times 10^{-7}$ & $\sigma_{\infty}, t_{B}=78 \%(1 / 2 \mathrm{Hr})$. \\
\hline \multirow[t]{2}{*}{528} & $5.43 \times 10^{-6}$ & $\sigma_{\mathrm{o}}$ \\
\hline & $1.62 \times 10^{-6}$ & $\sigma_{\infty}, t_{B}=70 \%(1 / 2 \mathrm{Hr})$. \\
\hline \multirow[t]{2}{*}{515} & $4.23 \times 10^{-6}$ & $\sigma_{\mathrm{o}}$ \\
\hline & $1.14 \times 10^{-6}$ & $\sigma_{\infty}, t_{B}=73 \%(1 / 2 \mathrm{Hr})$. \\
\hline 444 & $4.71 \times 10^{-6}$ & Cooling \\
\hline 410 & $6.25 \times 10^{-7}$ & Cooling \\
\hline \multirow[t]{2}{*}{383} & $3.30 \times 10^{-7}$ & $\sigma_{\mathrm{o}}$ \\
\hline & $8.19 \times 10^{-8}$ & $\sigma_{\infty}, t_{B}=75 \%(3 / 4 \mathrm{Hr})$. \\
\hline \multirow[t]{2}{*}{444} & $1.15 \times 10^{-6}$ & $\sigma_{\mathrm{o}}$ \\
\hline & $2.58 \times 10^{-7}$ & $\sigma_{\infty}, t_{B}=78 \%(3 / 4 \mathrm{Hr})$. \\
\hline \multirow[t]{2}{*}{476} & $2.08 \times 10^{-6}$ & $\sigma_{0}$ \\
\hline & $4.76 \times 10^{-7}$ & $\sigma_{\infty}, t_{B}=77 \%(1 / 2 \mathrm{Hr})$. \\
\hline \multirow[t]{2}{*}{405} & $5.08 \times 10^{-7}$ & $\sigma_{0}$ \\
\hline & $1.08 \times 10^{-7}$ & $\sigma_{\infty}, t_{B}=79 \%(1 \mathrm{Hr})$. \\
\hline
\end{tabular}


Table 8 (Contd.)

\begin{tabular}{cll}
$\begin{array}{c}\text { Temperature } \\
\left({ }^{\circ} \mathrm{C}\right)\end{array}$ & $\begin{array}{c}\text { Conductivity } \\
\left(\mathrm{ohm}^{-1} \mathrm{~cm}^{-1}\right)\end{array}$ & \multicolumn{1}{c}{ Remarks } \\
\hline 366 & $2.07 \times 10^{-7}$ & $\sigma_{\mathrm{O}}$ \\
& $4.63 \times 10^{-8}$ & $\sigma_{\infty}, t_{\mathrm{B}}=78 \%(1 \mathrm{Hr})$. \\
345 & $1.25 \times 10^{-7}$ & \\
294 & $3.22 \times 10^{-8}$ & \\
274 & $1.54 \times 10^{-8}$ & $\sigma_{\mathrm{O}}$ \\
& $6.57 \times 10^{-9}$ & $\sigma_{\infty}, t_{B}=43 \%(21 / 2 \mathrm{Hr})$. \\
\hline
\end{tabular}

\section{TABLE 9}

Data for jet milled polycrystalline sample presented in Figure 18

$$
t / A=0.416 \mathrm{~cm}^{-1}
$$

\begin{tabular}{cll}
$\begin{array}{c}\text { Temperature } \\
\left({ }^{\circ} \mathrm{C}\right)\end{array}$ & $\begin{array}{c}\text { Conductivity } \\
\left(0 \mathrm{hm}^{-1} \mathrm{~cm}^{-1}\right)\end{array}$ & Remarks \\
\hline 207 & $7.74 \times 10^{-9}$ & Heating \\
262 & $6.91 \times 10^{-8}$ & \\
290 & $1.54 \times 10^{-7}$ & \\
316 & 3.12 & Some polarization \\
371 & $1.01 \times 10^{-6}$ & \\
402 & 1.91 & \\
467 & 6.91 & \\
514 & $1.51 \times 10^{-5}$ & $\sigma_{0}$, overnight \\
423 & $2.65 \times 10^{-6}$ & $\sigma_{\infty}, t_{B}=91 \%(1 \mathrm{Hr})$. \\
& $2.50 \times 10^{-7}$ & $\sigma_{o}$ \\
473 & $6.91 \times 10^{-6}$ & $\sigma_{\infty}, t_{B}=86 \%(1 / 3 \mathrm{Hr})$.
\end{tabular}


l'able 9 (Contd.)

\begin{tabular}{|c|c|c|}
\hline $\begin{array}{c}\text { Temperature } \\
\left({ }^{\circ} \mathrm{C}\right)\end{array}$ & $\begin{array}{l}\text { Conductivity } \\
\left(0 h \mathrm{~m}^{-1} \mathrm{~cm}^{-1}\right)\end{array}$ & Remarks \\
\hline \multirow[t]{2}{*}{517} & $1.37 \times 10^{-5}$ & $\sigma_{0}$ \\
\hline & $1.75 \times 10^{-6}$ & $\sigma_{\infty}, t_{B}=87 \%(1 / 4 \mathrm{Hr})$. \\
\hline \multirow[t]{2}{*}{443} & $3.98 \times 10^{-6}$ & $\sigma_{\mathrm{o}}$ \\
\hline & $4.83 \times 10^{-7}$ & $\sigma_{\infty}, t_{B}=88 \%(1 / 4 \mathrm{Hr})$. \\
\hline \multirow[t]{2}{*}{415} & $2.20 \times 10^{-6}$ & $\sigma_{0}$ \\
\hline & $2.91 \times 10^{-7}$ & $\sigma_{\infty}, t_{B}=76 \%(2 / 3 \mathrm{Hr})$. \\
\hline \multirow[t]{2}{*}{520} & $1: 35 \times 10^{-5}$ & $\sigma_{\mathrm{o}}$ \\
\hline & $2.50 \times 10^{-6}$ & $\sigma_{\infty}, t_{B}=81 \%(1 / 2 \mathrm{Hr})$. \\
\hline \multirow[t]{2}{*}{542} & $1.85 \times 10^{-5}$ & $\sigma_{0}$ \\
\hline & $3.20 \times 10^{-6}$ & $\sigma_{\infty}, t_{B}=83 \%(1 / 3 \mathrm{Hr})$. \\
\hline \multirow[t]{2}{*}{452} & $4.53 \times 10^{-6}$ & $\sigma_{o}$, overnight \\
\hline & $5.57 \times 10^{-7}$ & $\sigma_{\infty}, t_{B}=88 \%(1 \mathrm{Hr})$. \\
\hline \multirow[t]{2}{*}{386} & $1.11 \times 10^{-6}$ & $\sigma_{\mathrm{o}}$ \\
\hline & $1.75 \times 10^{-7}$ & $\sigma_{\infty}, t_{B}=63 \%(1 \mathrm{Hr})$. \\
\hline \multirow[t]{2}{*}{333} & $3.02 \times 10^{-7}$ & $\sigma_{\mathrm{o}}$ \\
\hline & $7.03 \times 10^{-8}$ & $\sigma_{\infty}, t_{B}=77 \%(12 / 3$ Hrs. $)$ \\
\hline \multirow[t]{2}{*}{299} & $1.17 \times 10^{-7}$ & $\sigma_{o}$, overnight \\
\hline & $3.30 \times 10^{-8}$ & $\sigma_{\infty}, t_{B}=72 \%(12 / 3 \mathrm{Hrs})$. \\
\hline \multirow[t]{2}{*}{259} & $3.37 \times 10^{-8}$ & $\sigma_{\mathrm{o}}$ \\
\hline & $6.91 \times 10^{-9}$ & $\sigma_{\infty}, t_{B}=49 \%(21 / 2$ Hrs. $)$ \\
\hline
\end{tabular}


TABLE 10

Data for additively colored single crystal, short treatment, presented in Figure 21

$$
t / A=0.959 \mathrm{~cm}^{-1}
$$

\begin{tabular}{cll}
$\begin{array}{c}\text { Temperature } \\
\left({ }^{\circ} \mathrm{C}\right)\end{array}$ & $\begin{array}{c}\text { Conductivity } \\
(\text { ohm }\end{array}$ & Remarks \\
\hline 340 & $1.05 \times 10^{-8}$ & \\
402 & 3.33 & \\
450 & $1.10 \times 10^{-7}$ & \\
448 & $9.97 \times 10^{-8}$ & Overnight \\
487 & $3.38 \times 10^{-7}$ & \\
\hline
\end{tabular}

TABLE 11

Data for additively colored single crystal, medium treatment, presented in Figure 22

$$
t / A=0.652 \mathrm{~cm}^{-1}
$$

\begin{tabular}{cll}
$\begin{array}{c}\text { Temperature } \\
\left({ }^{\circ} \mathrm{C}\right)\end{array}$ & $\begin{array}{c}\text { Conductivity } \\
\left(\mathrm{ohm}^{-1} \mathrm{~cm}^{-1}\right)\end{array}$ & Remarks \\
\hline 367 & $2.54 \times 10^{-9}$ & Heating \\
402 & $1.10 \times 10^{-8}$ & \\
413 & 1.63 & \\
451 & 6.85 & 18 hour hold \\
467 & $1.26 \times 10^{-7}$ & $\sigma_{\mathrm{o}}$ \\
461 & $9.91 \times 10^{-8}$ & $\sigma_{\infty}, t_{\mathrm{B}}=16 \%(1 \mathrm{Hr})$. \\
457 & 8.41 & \\
479 & $1.90 \times 10^{-7}$ & \\
& $1.60 \times 10^{-7}$ &
\end{tabular}


Table 11 (Contd.)

\begin{tabular}{|c|c|c|}
\hline $\begin{array}{l}\text { Temperature } \\
\left.\qquad{ }^{\circ} \mathrm{C}\right)\end{array}$ & $\begin{array}{l}\text { Conductivity } \\
\left(\mathrm{ohm}^{-1} \mathrm{~cm}^{-1}\right)\end{array}$ & Remarks \\
\hline \multirow[t]{2}{*}{506} & $5.03 \times 10^{-7}$ & $\sigma_{o}$ \\
\hline & $3.95 \times 10^{-7}$ & $\sigma_{\infty}, t_{B}=21 \%(1 \mathrm{Hr})$. \\
\hline 540 & $1.58 \times 10^{-6}$ & Cooling \\
\hline 526 & 1.00 & \\
\hline \multirow[t]{2}{*}{518} & $7.37 \times 10^{-7}$ & $\sigma_{\mathrm{O}}$ \\
\hline & $5.71 \times 10^{-7}$ & $\sigma_{\infty}, t_{B}=22 \%(3 / 4 \mathrm{Hr})$. \\
\hline \multirow[t]{2}{*}{462} & $9.85 \times 10^{-8}$ & $\sigma_{0}$ \\
\hline & $8.48 \times 10^{-8}$ & $\sigma_{\infty}, t_{B}=14 \%(11 / 2$ Hrs. $)$ \\
\hline 380 & $3.56 \times 10^{-9}$ & Fast time polarization \\
\hline 304 & $1.16 \times 10^{-10}$ & \\
\hline 274 & $3.00 \times 10^{-11}$ & \\
\hline 264 & 1.70 & \\
\hline \multirow[t]{2}{*}{460} & $8.93 \times 10^{-8}$ & $\sigma_{0}$ \\
\hline & $7.95 \times 10^{-8}$ & $\sigma_{\infty}, t_{B}=11 \%(11 / 2 \mathrm{Hrs})$. \\
\hline \multirow[t]{2}{*}{510} & $5.83 \times 10^{-7}$ & $\sigma_{0}$ \\
\hline & $4.16 \times 10^{-7}$ & $\sigma_{\infty}, t_{B}=29 \%(1 \mathrm{Hr})$. \\
\hline \multirow[t]{2}{*}{532} & $1.15 \times 10^{-6}$ & $\sigma_{\mathrm{o}}$ \\
\hline & $8.41 \times 10^{-7}$ & $\sigma_{\infty}, t_{B}=26 \%(1 \mathrm{Hr})$. \\
\hline \multirow[t]{2}{*}{558} & $2.89 \times 10^{-6}$ & $\sigma_{\mathrm{o}}$ \\
\hline & $2.02 \times 10^{-6}$ & $\sigma_{\infty}, t_{B}=26 \%(3 / 4 \mathrm{Hr})$. \\
\hline
\end{tabular}


TABLE 12

Data for additively colored single crystal, long treatment, presented in Figure 23

$$
\mathrm{t} / \mathrm{A}=1.31 \mathrm{~cm}^{-1}
$$

\begin{tabular}{ll}
$\begin{array}{c}\text { Temperature } \\
\left({ }^{\circ} \mathrm{C}\right)\end{array}$ & $\begin{array}{l}\text { Conductivity } \\
\left(\text { ohm } \mathrm{cm}^{-1}\right)\end{array}$ \\
\hline 404 & $2.72 \times 10^{-7}$ \\
464 & $1.10 \times 10^{-6}$ \\
476 & 1.41 \\
456 & $9.03 \times 10^{-7}$ \\
426 & 4.69 \\
368 & $9.64 \times 10^{-8}$ \\
319 & 1.25 \\
276 & $2.69 \times 10^{-9}$ \\
294 & 5.34 \\
338 & $1.86 \times 10^{-8}$ \\
355 & 3.01 \\
376 & 5.67 \\
395 & $1.13 \times 10^{-7}$ \\
413 & 2.12 \\
409 & 1.53 \\
427 & 2.41 \\
505 & $2.20 \times 10^{-6}$ \\
459 & $9.41 \times 10^{-7}$ \\
409 & 3.14 \\
336 & $4.70 \times 10^{-8}$ \\
281 & $6.29 \times 10^{-9}$ \\
& 2.14 \\
&
\end{tabular}


Table 12 (Contd.)

\begin{tabular}{clc}
$\begin{array}{c}\text { Temperature } \\
\left({ }^{\circ} \mathrm{C}\right)\end{array}$ & $\begin{array}{c}\text { Conductivity } \\
(\text { ohm }\end{array}$ & Remarks \\
\hline 370 & $3.26 \times 10^{-8}$ & \\
410 & $1.36 \times 10^{-7}$ & 1 hour later \\
458 & 7.18 & 1 hou \\
459 & 7.96 &
\end{tabular}




\section{BIBLIOGRAPHY}

1. N. Mott and R. Gurney, Electronic Processes in Ionic Crystals, Dover Publications Inc., New York, 1964, pp. 270.

2. C. Tubandt, H. Reinhold, and G. Liebold, Z. anorg. u. allgem. Chem., 197, 225 (1931); Part III, Hdb. der. Exp. Physik., 12 [1] 383 (1932).

3. J. F. Laurent and J. Bénard, "Autodiffusion Des Ions Dans Les Halogemures Alcalius Polycristallius," J. Phys. Chem. Solids, 7, $218-27$ (1958).

4. J. Cabané, "Intergranular Diffusion and the Structure of Grain Boundaries in the Alkali Halides, Part 1 Method of the Study of Intergranular Diffusion Sintering; Part 2 Diffusion," J. Chem. Phys., 59, 1123-41 (1962).

5. A. B. Lidiard and K. Tharmalingam, "Diffusion Process at Low Temperatures, " Discussion Faraday Soc., 56, 64-68 (1960).

6. N. Laurance, "Self-Diffusion of the Chloride Ion in Sodium Chloride," Phys. Rev., 120 [1] 57-62 (1960).

7. L. W. Barr, J. A. Morrison, and P. A. Schroeder, "Anion Diffusion in Crystals of $\mathrm{NaCl}, " \mathrm{~J}$. of Appl. Phys., 36 [2] 624-31 (1965).

8. A. B. Lidiard, "Ionic Conductivity," Hdb. der Physik, 20, 246-349 (1957).

9. R. W. Dreyfus and A. S. Nowick, "Ionic Conductivity of Doped $\mathrm{NaCl}$ Crystals, " Phys. Rev., 126 [4] 1367-77 (1962).

10. R. W. Dreyfus and A. S. Nowick, "Energy and Eutropy of Formation and Motion of Vacancies in $\mathrm{NaCl}$ and $\mathrm{KCl}$ Crystals," J. Appl. Phys., Supplement to 33 [1] 473-77 (1962). 
11. F. G. Fumi and M. P. Tosi, "Lattice Calculations on Point Imperfections in the Alkali Halides, "Discussions Faraday Soc., 23, $92-98(1957)$.

12. L. W. Barr, I. M. Hoodless, J. A. Morrison, and R. Rudham, "Effects of Gross Imperfections on Chloride Ion Diffusion in Crystals of Sodium Chloride and Potassium Chloride, "Trans. Faraday Soc. , 56, 697-708 (1960).

13. J. A. Morrison and R. Rudham, "Anion Diffusion in Doped Crystals of Potassium Chloride," J. Phys. Chem. Solids, 6, 402-404 (1958).

14. F. Seitz, "Color Centers in Alkali Halide Crystals," Revs. Modern Phys., 18, 384-408 (1946).

15. M. P. Tosi and F. G. Fumi, "The Interaction between Equilibrium Defects in the Alkali Halides: the 'Ground State' Binding Energy of the Vacancy Pair," Nuovo cimento, 7 [1] 95-106 (1958).

16. F. A. Kroger and H. J. Vink, "Relations between the Concen trations of Imperfections in Crystalline Solids, "Solid State Physics, Academic Press Inc., New York, 3, 310-431 (1956).

17. J. N. Maycock, "Electrical Effects due to the F-Center in Potassium Halides, " J. Appl. Phys., 35 [5] 1512-15 (1964).

18. J. H. de Boer, Recwil Trav. Chim. Pays-Bas, 56, 301 (1937).

19. J. H. Schulman and W. D. Compton, Color Centers in Solids, p. 93, The Macmillan Co, , New York, 1963, 368 pp.

20. R. W. Pohl, "Electron Conductivity and Photoelectric Processes" Proc. Phys. Soc. (London) 49 (extra part) [3] (1937).

21. J. Hokkinson, Phil. Trans. Roy Soc. (London) 166, 489 (1876):

J. and P. Curie, Am. Chem. Phys., 17, $385(\overline{1888})$.

22. R. W. Dreyfus, "Dielectric Relaxation Due to Impurity-Vacancy Complexes in NaCl Crystals," Phys. Rev., 121 [6] 1675-87 (1961).

23. R. W. Dreyfus and R. B. Laibowitz, "Anelastic and Dielectric Relaxation due to Impurity-Vacancy Complexes in NaCl Crystals, " Phys. Rev., 135 [5A] A 1413-22 (1964). 
24. P. H. Sutter and A. S. Nowick, "Ionic Conductivity and Time Dependent Polarization in $\mathrm{NaCl}$ Crystals, " J. Appl. Phys., 34 [4] (Part 1) 734-46 (1963).

25. P. W. M. Jacobs and J. N. Maycock, "Polarization Effects in the Ionic Conductivity of Alkali Halide Crystals. I A. C. Capacity," J. Chem. Phys., 39 [3] 757-62 (1963).

26. N. M. Tallan and J. M. Wimmer, unpublished private communication.

27. N. M. Tallan and H. C. Graham, "Interfacial Polarization and Electrical Conductivity in Sapphire," to appear in J. Am. Ceram. Soc.

28. A. R. Allnatt and P. W. M. Jacobs, "Conductance of Potassium Chloride in the Solid State, "Trans. Faraday Soc., 58, 116-31 (1962).

29. A. R. Allnatt and P. W. M. Jacobs, "A. C. Polarization Effects in the Ionic Conductivity of Potassium Chloride Crystals, " J. Phys. Chem. Solids, 19, 281-90 (1961).

30. R. W. Vest, "Apparatus for Guarded Polarization Measurements," Submitted for publication to Rev. of Sci. Inst.

31. Biron, J. of Russian Phys. - Chem. Soc. (Chem. Part) 40, 1609 (1908); International Critical Tables III, 28, 33 (1928).

32. K. Lekovic, "Space-Charge Layer and Distribution of Lattice Defects at the Surface of Ionic Crystals," J. Chem. Phys., 21 [7] $1123-28$ (1953).

33. R. Guccione, M. P. Tosi, and M. Asdente, "Migration Barriers for Cations and Anions in Alkali Halide Crystals," J. Phys. Chem. Solids, 10, 162-68 (1959).

34. G. Brouwer, Philips Research Repts., 9, 366 (1954). 